The Astrophysical Journal, 689:499-512, 2008 December 10

(C) 2008. The American Astronomical Society. All rights reserved. Printed in U.S.A.

\title{
ANALYTIC APPROXIMATIONS FOR TRANSIT LIGHT-CURVE OBSERVABLES, UNCERTAINTIES, AND COVARIANCES
}

\author{
Joshua A. Carter, ${ }^{1}$ Jennifer C. Yee, ${ }^{2}$ Jason Eastman, $^{2}$ B. Scott Gaudi, ${ }^{2}$ and Joshua N. Winn ${ }^{1}$ \\ Received 2008 May 2; accepted 2008 August 1
}

\begin{abstract}
The light curve of an exoplanetary transit can be used to estimate the planetary radius and other parameters of interest. Because accurate parameter estimation is a nonanalytic and computationally intensive problem, it is often useful to have analytic approximations for the parameters as well as their uncertainties and covariances. Here, we give such formulae, for the case of an exoplanet transiting a star with a uniform brightness distribution. We also assess the advantages of some relatively uncorrelated parameter sets for fitting actual data. When limb darkening is significant, our parameter sets are still useful, although our analytic formulae underpredict the covariances and uncertainties.
\end{abstract}

Subject headings: binaries: eclipsing — methods: analytical — planets and satellites: general

\section{INTRODUCTION}

The transit of an exoplanet across the face of its parent star is an opportunity to learn a great deal about the planetary system. Photometric and spectroscopic observations reveal details about the planetary radius, mass, atmosphere, and orbit, as reviewed recently by Charbonneau et al. (2007). Transit light curves, in particular, bear information about the planetary and stellar radii, the orbital inclination, and the mean density of the star (Mandel \& Agol 2002; Seager \& Mallén-Ornelas 2003; Giménez 2007). Additional planets in the system may be detected through gradual changes in the orbital parameters of the transiting planet (MiraldaEscudé 2002; Heyl \& Gladman 2007) or from a pattern of anomalies in a collection of midtransit times (Holman \& Murray 2005; Agol et al. 2005; Ford \& Holman 2007).

In general, the parameters of a transiting system and their uncertainties must be estimated from the photometric data using numerical methods. For example, many investigators have used $\chi^{2}$-minimization schemes such as AMOEBA or the LevenbergMarquardt method, along with confidence levels determined by examining the appropriate surface of constant $\Delta \chi^{2}$ (see, e.g., Brown et al. 2001; Alonso et al. 2004) or by bootstrap methods (e.g., Sato et al. 2005; Winn et al. 2005). More recently, it has become common to use Markov chain Monte Carlo (MCMC) methods (e.g., Holman et al. 2006; Winn et al. 2007; Burke et al. 2007). However, even when numerical algorithms are required for precise answers, it is often useful to have analytic approximations for the parameters as well as their uncertainties and covariances.

Analytic approximations can be useful for planning observations. For example, one may obtain quick answers to questions such as, for which systems can I expect to obtain the most precise measurement of the orbital inclination? Or, how many transit light curves will I need to gather with a particular telescope before the statistical error in the planetary radius is smaller than the systematic error? Now that nearly 50 transiting planets are known, we enjoy a situation in which a given night frequently offers more than one observable transit event. Analytic calculations can help one decide which target is more fruitfully observed, and are much simpler and quicker than the alternative of full numerical sim-

\footnotetext{
${ }^{1}$ Department of Physics and Kavli Institute for Astrophysics and Space Research, Massachusetts Institute of Technology, Cambridge, MA 02139.

${ }^{2}$ Department of Astronomy, Ohio State University, 140 West 18th Avenue, Columbus, OH 43210.
}

ulations. Analytic approximations are also useful for understanding the parameter degeneracies inherent in the model and for constructing relatively uncorrelated parameter sets that will speed the convergence of optimization algorithms. Finally, analytic approximations are useful in order-of-magnitude estimates of the observability of subtle transit effects, such as transit timing variations, precession-induced changes in the transit duration, or the asymmetry in the ingress and egress durations due to a nonzero orbital eccentricity.

Mandel \& Agol (2002) and Giménez (2007) have previously given analytic formulae for the received flux as a function of the relative separation of the planet and the star, but their aim was to provide highly accurate formulae, which are too complex for useful analytic estimates of uncertainties and covariances. Protopapas et al. (2005) provided an analytic and differentiable approximation to the transit light curve, but they were concerned with speeding up the process of searching for transits in large databases, rather than parameter estimation. Seager \& Mallén-Ornelas (2003) presented an approximate model of a transit light curve with the desired level of simplicity, but did not provide analytic estimates of uncertainties and covariances.

This paper is organized as follows. In $\S 2$ we present a simple analytic model for a transit light curve, using a convenient and intuitive parameterization similar to that of Seager \& MallénOrnelas (2003). In $\S 3$ we derive analytic approximations for the uncertainties and covariances of the basic parameters, and in $\S 4$ we verify the accuracy of those approximations through numerical tests. Our model assumes that the flux measurements are made continuously throughout the transit and that stellar limb darkening is negligible; in $\S \S 4.1$ and 4.3 we check on the effects of relaxing these assumptions. In $\S 5$ we derive some useful expressions for the uncertainties in some especially interesting or useful "derived" parameters, i.e., functions of the basic model parameters. In $\S 6$ we present alternative parameter sets that are better suited to numerical algorithms for parameter estimation utilizing the analytic formalism given in $\S 3$. We compare the correlations among parameters for various parameter sets that have been used in the transit literature. Finally, $\S 7$ gives a summary of the key results.

\section{LINEAR APPROXIMATION TO THE TRANSIT LIGHT CURVE}

Imagine a spherical star of radius $R_{\star}$ with a uniform brightness and an unocculted flux $f_{0}$. When a dark, opaque, spherical planet of radius $R_{p}$ is in front of the star, at a center-to-center 
sky-projected distance of $z R_{\star}$, the received stellar flux is $F^{e}\left(r, z, f_{0}\right)=f_{0}\left[1-\lambda^{e}(r, z)\right]$, where

$$
\begin{aligned}
& \lambda^{e}(r, z) \\
& = \begin{cases}0, & 1+r<z, \\
\frac{1}{\pi}\left[r^{2} \kappa_{0}+\kappa_{1}-\sqrt{\frac{4 z^{2}-\left(1+z^{2}-r^{2}\right)^{2}}{4}}\right], & 1-r<z \leq 1+r, \\
r^{2}, & z \leq 1-r,\end{cases}
\end{aligned}
$$

with $\kappa_{1}=\cos ^{-1}\left[\left(1-r^{2}+z^{2}\right) / 2 z\right]$ and $\kappa_{0}=\cos ^{-1}\left[\left(r^{2}+z^{2}-\right.\right.$ 1)/2rz] (Mandel \& Agol 2002). Geometrically, $\lambda^{e}$ is the overlap area between two circles with radii 1 and $r$ whose centers are $z$ units apart. The approximation of uniform brightness (no limb darkening) is valid for mid-infrared bandpasses, which are increasingly being used for transit observations (see, e.g., Richardson et al. 2006; Knutson et al. 2007; Deming et al. 2007), and is a good approximation even for near-infrared and far-red bandpasses. We make this approximation throughout this paper, except in $\S 4.3$ where we consider the effect of limb darkening.

For a planet on a circular orbit, the relation between $z$ and the time $t$ is

$$
z(t)=a R_{\star}^{-1} \sqrt{\left[\sin n\left(t-t_{c}\right)\right]^{2}+\left[\cos i \cos n\left(t-t_{c}\right)\right]^{2}},
$$

where $a$ is the semimajor axis, $i$ is the inclination angle, $n \equiv 2 \pi / P$ is the angular frequency with period $P$, and $t_{c}$ is the transit midpoint (when $z$ is smallest).

The four "contact times" of the transit are the moments when the planetary disk and stellar disk are tangent. First contact $\left(t_{\mathrm{I}}\right)$ occurs at the beginning of the transit, when the disks are externally tangent. Second contact $\left(t_{\mathrm{II}}\right)$ occurs next, when the disks are internally tangent. Third and fourth contacts $\left(t_{\mathrm{III}}\right.$ and $\left.t_{\mathrm{IV}}\right)$ are the moments of internal and external tangency, respectively, as the planetary disk leaves the stellar disk. The total transit duration is $t_{\mathrm{IV}}-t_{\mathrm{I}}$. The ingress phase is defined as the interval between $t_{\mathrm{I}}$ and $t_{\mathrm{II}}$, and likewise, the egress phase is defined as the interval between $t_{\mathrm{III}}$ and $t_{\mathrm{IV}}$. We also find it useful to define the ingress midpoint $t_{\text {ing }} \equiv\left(t_{\mathrm{I}}+t_{\mathrm{II}}\right) / 2$ and the egress midpoint $t_{\mathrm{egr}} \equiv\left(t_{\mathrm{III}}+t_{\mathrm{IV}}\right) / 2$.

Although equations (1) and (2) give an exact solution, they are too complicated for an analytic error analysis. We make a few approximations to enable such an analysis. First, we assume the orbital period is large compared to the transit duration, in which case equation (2) is well approximated by

$$
z(t)=\sqrt{\left[\left(t-t_{c}\right) / \tau_{0}\right]^{2}+b^{2}},
$$

where for a circular orbit, $\tau_{0}=R_{\star} P / 2 \pi a=R_{\star} / n a$ and $b=$ $a \cos i / R_{\star}$ is the normalized impact parameter. In this limit, the planet moves uniformly in a straight line across the stellar disk. Simple expressions may be derived for two characteristic timescales of the transit,

$$
\begin{aligned}
t_{\text {egr }}-t_{\text {ing }} & =\tau_{0}\left[\sqrt{(1+r)^{2}-b^{2}}+\sqrt{(1-r)^{2}-b^{2}}\right] \\
& =2 \tau_{0} \sqrt{1-b^{2}}+O\left(r^{2}\right), \\
t_{\mathrm{II}}-t_{\mathrm{I}} & =\tau_{0}\left[\sqrt{(1+r)^{2}-b^{2}}-\sqrt{(1-r)^{2}-b^{2}}\right] \\
& =2 \tau_{0} \frac{r}{\sqrt{1-b^{2}}}+O\left(r^{3}\right) .
\end{aligned}
$$

Flux

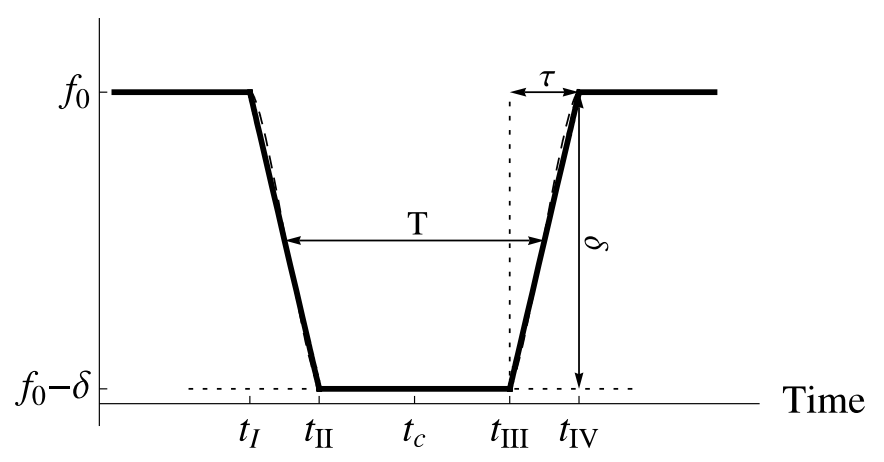

FIG. 1.-Comparison of the exact and piecewise linear transit models, for the parameter choice $r=0.2$ and $b=0.5$. The dashed line shows the exact uniformsource model $F^{e}$, given by eq. (1). The solid line shows the linear model $F^{l}$, given by eq. (11).

It is easy to enlarge the discussion to include eccentric orbits, by replacing $a$ by the planet-star distance at midtransit and $n$ by the angular frequency at midtransit,

$$
\begin{gathered}
a \rightarrow \frac{a\left(1-e^{2}\right)}{1+e \sin \omega}, \\
n \rightarrow \frac{n(1+e \sin \omega)^{2}}{\left(1-e^{2}\right)^{3 / 2}},
\end{gathered}
$$

where $e$ is the eccentricity, and $\omega$ is the argument of pericenter. Here, too, we approximate the planet's actual motion by uniform motion across the stellar disk, with a velocity equal to the actual velocity at midtransit. Methods for computing these quantities at midtransit are discussed by Murray \& Dermott (2000) as well as in recent transit-specific studies by Barnes (2007), Burke (2008), Ford et al. (2008), and Gillon et al. (2007). We redefine the parameters $\tau_{0}$ and $b$ in this expanded scope as

$$
\begin{gathered}
b \equiv \frac{a \cos i}{R_{\star}}\left(\frac{1-e^{2}}{1+e \sin \omega}\right), \\
\tau_{0} \equiv \frac{R_{\star}}{a n}\left(\frac{\sqrt{1-e^{2}}}{1+e \sin \omega}\right) .
\end{gathered}
$$

We do not restrict our discussion to circular orbits $(e=0)$ unless otherwise stated.

Next, we replace the actual light curve with a model that is piecewise linear in time, as illustrated in Figure 1. Specifically, we define the parameters

$$
\begin{gathered}
\delta \equiv f_{0} r^{2}=f_{0}\left(R_{p} / R_{\star}\right)^{2}, \\
T \equiv 2 \tau_{0} \sqrt{1-b^{2}}, \\
\tau \equiv 2 \tau_{0} \frac{r}{\sqrt{1-b^{2}}},
\end{gathered}
$$

and then we define our model light curve as

$$
\begin{aligned}
& F^{l}(t) \\
& = \begin{cases}f_{0}-\delta, & \left|t-t_{c}\right| \leq T / 2-\tau / 2, \\
f_{0}-\delta+(\delta / \tau) & \\
\quad \times\left(\left|t-t_{c}\right|-T / 2+\tau / 2\right), & T / 2-\tau / 2<\left|t-t_{c}\right|<T / 2+\tau / 2, \\
f_{0}, & \left|t-t_{c}\right| \geq T / 2+\tau / 2,\end{cases}
\end{aligned}
$$


We use the symbol $F^{l}$ to distinguish this piecewise linear model ( $l$ for linear) from the exact uniform-source expression $F^{e}$ given by equations (1) and (2). The deviations between $F^{l}$ and $F^{e}$ occur near and during the ingress and egress phases. The approximation is most accurate in the limit of small $r$ and $b$ and is least accurate for grazing transits. As shown in equation (5), when $r$ is small, $\tau \approx t_{\mathrm{II}}-t_{\mathrm{I}}$ (the ingress or egress duration) and $T \approx$ $t_{\mathrm{egr}}-t_{\text {ing }}$ (the total transit duration). Neither this piecewise linear model nor the choice of parameters is new. Seager \& MallénOrnelas (2003) also used a piecewise linear model, with different linear combinations of these parameters, and both Burke et al. (2007) and Bakos et al. (2007) have employed parameterizations that are closely related to the parameters given above. What is specifically new to this paper is an analytic error and covariance analysis of this linear model, along with useful analytic expressions for errors in the physical parameters of the system. The "inverse" mapping from our parameterization to a more physical parameterization is

$$
\begin{gathered}
r^{2}=\left(R_{p} / R_{\star}\right)^{2}=\delta / f_{0}, \\
b^{2}=\left(\frac{a \cos i}{R_{\star}}\right)^{2}\left(\frac{1-e^{2}}{1+e \sin \omega}\right)^{2}=1-r \frac{T}{\tau}, \\
\tau_{0}^{2}=\left(\frac{R_{\star}}{a n}\right)^{2}\left(\frac{\sqrt{1-e^{2}}}{1+e \sin \omega}\right)^{2}=\frac{T \tau}{4 r} .
\end{gathered}
$$

\section{FISHER INFORMATION ANALYSIS}

Given a model $F\left(t ;\left\{p_{i}\right\}\right)$ with independent variable $t$ and a set of parameters $\left\{p_{i}\right\}$, it is possible to estimate the covariance between parameters, $\operatorname{Cov}\left(p_{i}, p_{j}\right)$, that would be obtained by measuring $F(t)$ with some specified cadence and precision. (Gould [2003] gives a pedagogical introduction to this technique.) Suppose we have $N$ data points taken at times $t_{k}$ spanning the entire transit event. The error in each data point is assumed to be a Gaussian random variable, with zero mean and standard deviation $\sigma_{k}$. Then the covariance between parameters $\left\{p_{i}\right\}$ is

$$
\operatorname{Cov}\left(p_{i}, p_{j}\right)=\left(B^{-1}\right)_{i j},
$$

where $\mathbf{B}$ is the zero-mean Gaussian noise Fisher information matrix, which is calculated as

$$
B_{i j}=\sum_{k=1}^{N} \sum_{l=1}^{N}\left[\frac{\partial}{\partial p_{i}} F\left(t_{k} ;\left\{p_{m}\right\}\right)\right] \mathcal{B}_{k l}\left[\frac{\partial}{\partial p_{j}} F\left(t_{l} ;\left\{p_{m}\right\}\right)\right] .
$$

Here, $\mathcal{B}_{k l}$ is the inverse covariance matrix of the flux measurements. We assume the measurement errors are uncorrelated (i.e., we neglect "red noise"), in which case $\mathcal{B}_{k l}=\delta_{k l} \sigma_{k}^{-2}$. We further assume that the measurement errors are uniform in time with $\sigma_{k}=\sigma$, giving $\mathcal{B}_{k l}=\delta_{k l} \sigma^{-2}$.

In Table 1 we compute the needed partial derivatives ${ }^{3}$ of the piecewise linear light curve $F^{l}$, which has five parameters $\left\{p_{i}\right\}=\left\{t_{c}, \tau, T, \delta, f_{0}\right\}$.

Figure 2 shows the time dependence of the parameter derivatives, for a particular case. The time dependence of the parameter derivatives for the exact uniform-source model $F^{e}$ is also shown, for comparison, as are the numerical derivatives for limb-darkened light curves. This comparison shows that the linear

\footnotetext{
${ }^{3}$ In computing these derivatives we have ignored the dependence of the piecewise boundaries in Table 1 on the parameter values. The derivatives associated with those boundary changes are finite and have a domain of measure zero in the limit of continuous sampling. Thus, they do not affect our covariance calculation.
}

TABLE 1

Partial Derivatives of the Piecewise Linear Light Curve $F^{l}$, IN the Five Parameters $p_{i}=\left\{t_{c}, \tau, T, \delta, f_{0}\right\}$

\begin{tabular}{cccc}
\hline \hline Partial Derivative & Totality & Ingress/Egress & Out of Transit \\
\hline$\left(\partial / \partial t_{c}\right) F^{l}\left(t ; p_{m}\right) \ldots \ldots .$. & 0 & $-(\delta / \tau)\left(t-t_{c}\right) /\left|t-t_{c}\right|$ & 0 \\
$(\partial / \partial \tau) F^{l}\left(t ; p_{m}\right) \ldots \ldots \ldots$. & 0 & $-\left(\delta / \tau^{2}\right)\left[\left|t-t_{c}\right|-(T / 2)\right]$ & 0 \\
$(\partial / \partial T) F^{l}\left(t ; p_{m}\right) \ldots \ldots .$. & 0 & $-\delta /(2 \tau)$ & 0 \\
$(\partial / \partial \delta) F^{l}\left(t ; p_{m}\right) \ldots \ldots \ldots$. & -1 & $(1 / \tau)\left[\left|t-t_{c}\right|-(T / 2)\right]-1 / 2$ & 0 \\
$\left(\partial / \partial f_{0}\right) F^{l}\left(t ; p_{m}\right) \ldots \ldots .$. & 1 & 1 & 1 \\
\hline
\end{tabular}

Note.-The intervals $\left|t-t_{c}\right|<T / 2-\tau / 2, T / 2-\tau / 2<\left|t-t_{c}\right|<T / 2+\tau / 2$, and $\left|t-t_{c}\right|>T / 2+\tau / 2$ correspond to totality, ingress/egress, and out of transit, respectively.

model captures the essential features of more realistic models and, in particular, the symmetries. The most obvious problem with the linear model is that it gives a poor description of the $\tau$-derivative and the $\delta$-derivative for the case of appreciable limb darkening, as discussed further in $\S 4.3$. From Figure 2 and Table 1 we see that for the parameters $T, \tau$, and $\delta$, the derivatives are symmetric about $t=t_{c}$, while the derivative for the parameter $t_{c}$ is antisymmetric about $t_{c}$. This implies that $t_{c}$ is uncorrelated with the other parameters. (This is also the case for the exact model, with or without limb darkening.)

We suppose that the data points are sampled uniformly in time at a rate $\Gamma=N / T_{\text {tot }}$, where the observations range from $t=t_{0}$ to $t=t_{0}+T_{\text {tot }}$ and encompass the entire transit event. In the limit of large $\Gamma \tau$ we may approximate the sums of equation (16) with time integrals,

$$
B_{i j}=\frac{\Gamma}{\sigma^{2}} \int_{t_{0}}^{t_{0}+T_{\text {tot }}}\left[\frac{\partial}{\partial p_{i}} F^{l}\left(t ;\left\{p_{m}\right\}\right)\right]\left[\frac{\partial}{\partial p_{j}} F^{l}\left(t ;\left\{p_{m}\right\}\right)\right] d t .
$$

Using the derivatives from Table 1 we find

$$
B=\frac{\Gamma}{\sigma^{2}}\left(\begin{array}{ccccc}
\frac{2 \delta^{2}}{\tau} & 0 & 0 & 0 & 0 \\
0 & \frac{\delta^{2}}{6 \tau} & 0 & -\frac{\delta}{6} & 0 \\
0 & 0 & \frac{\delta^{2}}{2 \tau} & \frac{\delta}{2} & -\delta \\
0 & -\frac{\delta}{6} & \frac{\delta}{2} & T-\frac{\tau}{3} & -T \\
0 & 0 & -\delta & -T & T_{\text {tot }}
\end{array}\right) .
$$

In what follows, it is useful to define some dimensionless variables,

$$
\begin{gathered}
Q \equiv \sqrt{\Gamma T} \frac{\delta}{\sigma}, \\
\theta \equiv \tau / T, \\
\eta \equiv T /\left(T_{\text {tot }}-T-\tau\right) .
\end{gathered}
$$

The first of these variables, $Q$, is equal to the total signal-tonoise ratio of the transit in the limit $r \rightarrow 0$. The second variable, $\theta$, is approximately the ratio of ingress (or egress) duration to the total transit duration. The third variable, $\eta$, is approximately the ratio of the number of data points obtained during the transit to the number of data points obtained before or after the transit. Oftentimes, $r$ and $\theta$ are much smaller than unity, which will later enable us to derive simple expressions for the variances and covariances, but for the moment we consider the general case. 


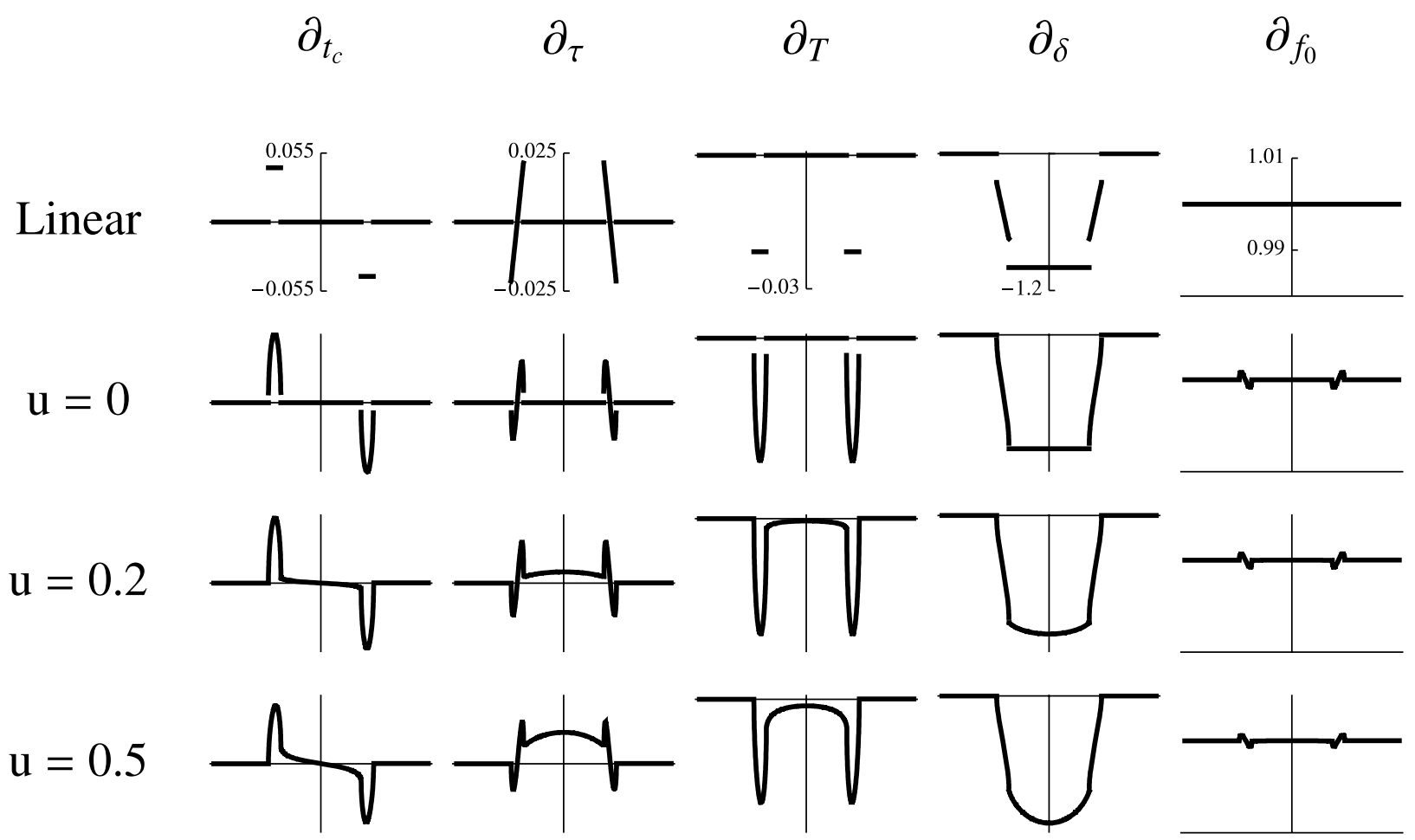

FIG. 2.-Parameter derivatives, as a function of time, for the piecewise linear model light curve $F^{l}$ (top row), the exact light curve for the case of zero limb darkening

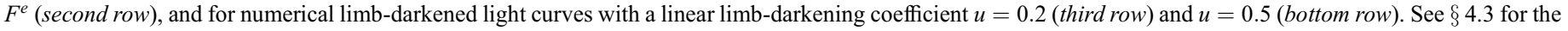
definition of $u$. Typical scales are shown in the first row and are consistent in the following rows.

Inverting $\mathbf{B}$, we find the covariance matrix for the piecewise linear model,

$$
\begin{aligned}
& \operatorname{Cov}\left(\left\{t_{c}, \tau, T, \delta, f_{0}\right\},\left\{t_{c}, \tau, T, \delta, f_{0}\right\}\right)= \\
& \frac{1}{Q^{2}}\left(\begin{array}{ccccc}
\frac{\theta}{2} T^{2} & 0 & 0 & 0 & 0 \\
0 & a \theta T^{2} & b \theta^{2} T^{2} & c \theta \delta T & \eta \theta \delta T \\
0 & b \theta^{2} T^{2} & d \theta T^{2} & b \theta \delta T & \eta \theta \delta T \\
0 & c \theta \delta T & b \theta \delta T & c \delta^{2} & \eta \delta^{2} \\
0 & \eta \theta \delta T & \eta \theta \delta T & \eta \delta^{2} & \eta \delta^{2}
\end{array}\right),
\end{aligned}
$$

where $a=\eta \theta+(6-5 \theta) /(1-\theta), b=\eta-1 /(1-\theta), c=\eta+$ $1 /(1-\theta)$, and $d=\eta \theta+(2-\theta) /(1-\theta)$. The elements along the diagonal of the covariance matrix are variances, or squares of standard errors, $\sigma_{p_{i}}=\left[\operatorname{Cov}\left(p_{i}, p_{i}\right)\right]^{1 / 2}$.

This result can be simplified for the case when many out-oftransit observations are obtained and $\eta \rightarrow 0$. In this limit, $f_{0}$ is known with negligible error, and we may assume $f_{0}=1$ without loss of generality. In this case, $\delta$ is the fractional transit depth, and the covariance matrix becomes

$$
\begin{aligned}
& \operatorname{Cov}\left(\left\{t_{c}, \tau, T, \delta\right\},\left\{t_{c}, \tau, T, \delta\right\}\right)= \\
& \frac{1}{Q^{2}}\left(\begin{array}{cccc}
\frac{\theta}{2} T^{2} & 0 & 0 & 0 \\
0 & \frac{\theta(6-5 \theta)}{1-\theta} T^{2} & -\frac{\theta^{2}}{1-\theta} T^{2} & \frac{\theta}{1-\theta} \delta T \\
0 & -\frac{\theta^{2}}{1-\theta} T^{2} & \frac{\theta(2-\theta)}{1-\theta} T^{2} & -\frac{\theta}{1-\theta} \delta T \\
0 & \frac{\theta}{1-\theta} \delta T & -\frac{\theta}{1-\theta} \delta T & \frac{1}{1-\theta} \delta^{2}
\end{array}\right) .
\end{aligned}
$$

from which it is obvious that $\theta$ is the key controlling parameter that deserves special attention. Using equations (9) and (10) we may write

$$
\theta=\frac{r}{1-b^{2}}
$$

Unless the transit is grazing, we have $b \leq 1-r$, and $\theta$ is restricted to the range $[r, 1 /(2-r)]$. Figure 3 shows the dependence of $\theta$ on the impact parameter, for various choices of the transit depth. It is important to keep in mind that for $b \lesssim 0.5, \theta$ is nearly equal to $r$ and depends weakly on $b$. This implies that $\theta$ is expected to be quite small for most transiting systems. For planetary orbits that are randomly oriented in space, the expected distribution of $b$ is uniform, and hence, we expect $\theta \lesssim 0.3$ for

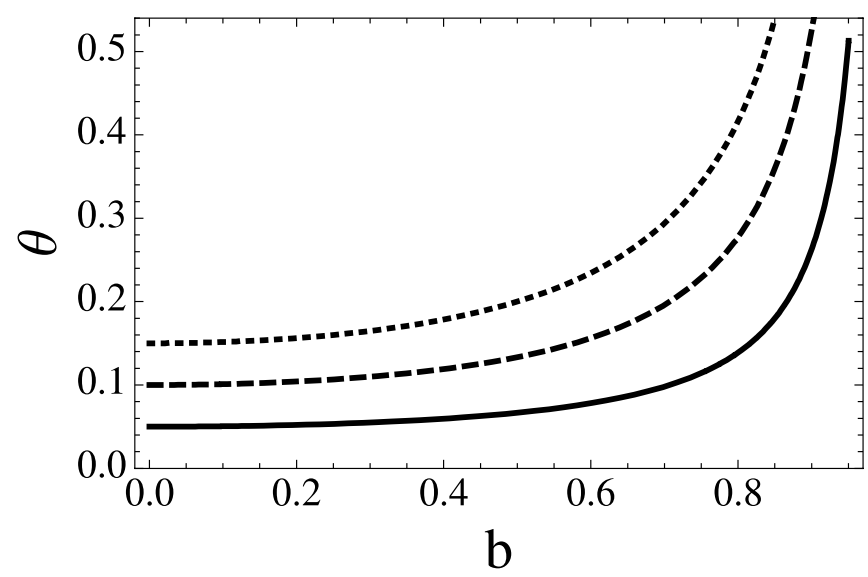

FIG. 3.-Dependence of $\theta=\tau / T$ on depth $\delta=r^{2}$ and normalized impact parameter $b$, for the cases $r=0.05$ (solid line), 0.1 (dashed line), and 0.15 (dotted line). 

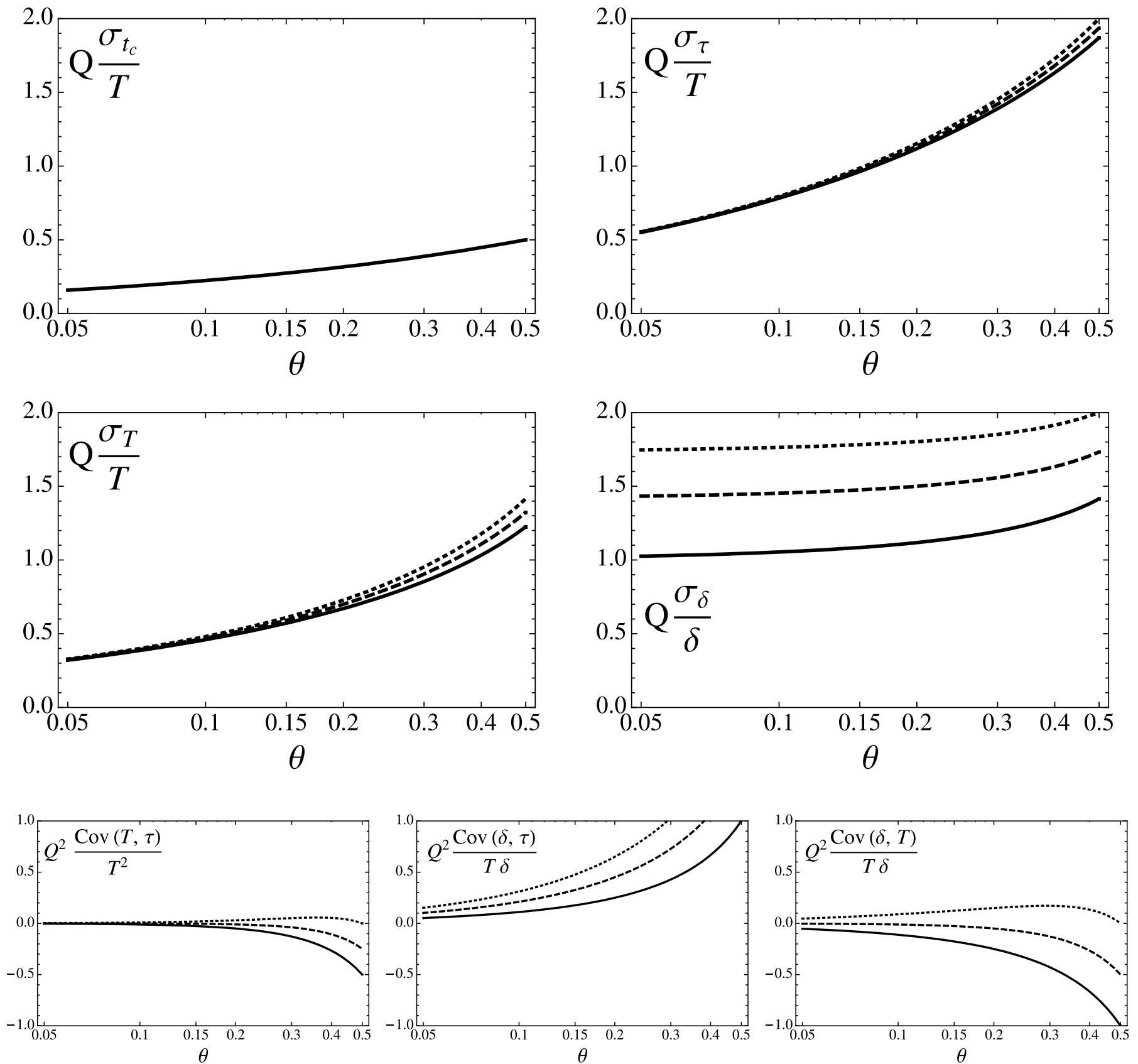

FIG. 4.-Standard errors and covariances, as a function of $\theta \equiv \tau / T$, for different choices of $\eta$. The analytic expressions are given in eq. (20). The definitions of $\eta, \theta$, and $Q$ are given in eq. (19). We show results for $\eta=0$ (solid lines), $\eta=0.5$ (dashed lines), and $\eta=1$ (dotted lines).

$90 \%$ of a random sample of transiting planets with $R_{p} \leq R_{\mathrm{J}}{ }^{4}$ For this reason, in the following figures we use a logarithmic scale for $\theta$, to emphasize the small values. Figure 4 shows the (suitably normalized) elements of the covariance matrix as a function of $\theta$.

In the limits $\eta \rightarrow 0$ (errorless knowledge of $f_{0}$ ) and $\theta \rightarrow r$ (small impact parameter), the expressions for the standard errors are especially simple,

$$
\begin{gathered}
\sigma_{t_{c}}=Q^{-1} T \sqrt{\theta / 2}, \\
\sigma_{\tau} \approx Q^{-1} T \sqrt{6 \theta},
\end{gathered}
$$

\footnotetext{
${ }^{4}$ In fact, the fraction of discovered systems with $\theta \lesssim 0.3$ may be even larger than $90 \%$, because selection effects make it harder to detect grazing transits.
}

$$
\begin{gathered}
\sigma_{T} \approx Q^{-1} T \sqrt{2 \theta}, \\
\sigma_{\delta} \approx Q^{-1} \delta .
\end{gathered}
$$

In this regime, we have a clear hierarchy in the precision with which the time parameters are known, with $\sigma_{t_{c}}<\sigma_{T}<\sigma_{\tau}$.

To further quantify the degree of correlation among the parameters, we compute the correlation matrix,

$$
\begin{aligned}
& \operatorname{Corr}\left(\left\{t_{c}, \tau, T, \delta, f_{0}\right\},\left\{t_{c}, \tau, T, \delta, f_{0}\right\}\right) \\
& =\frac{\operatorname{Cov}(i, j)}{\sqrt{\operatorname{Cov}(i, i) \operatorname{Cov}(j, j)}}=\left(\begin{array}{llllll}
1 & 0 & 0 & 0 & 0 \\
0 & 1 & a & b & c \\
0 & a & 1 & d & e \\
0 & b & d & 1 & f \\
0 & c & e & f & 1
\end{array}\right),
\end{aligned}
$$



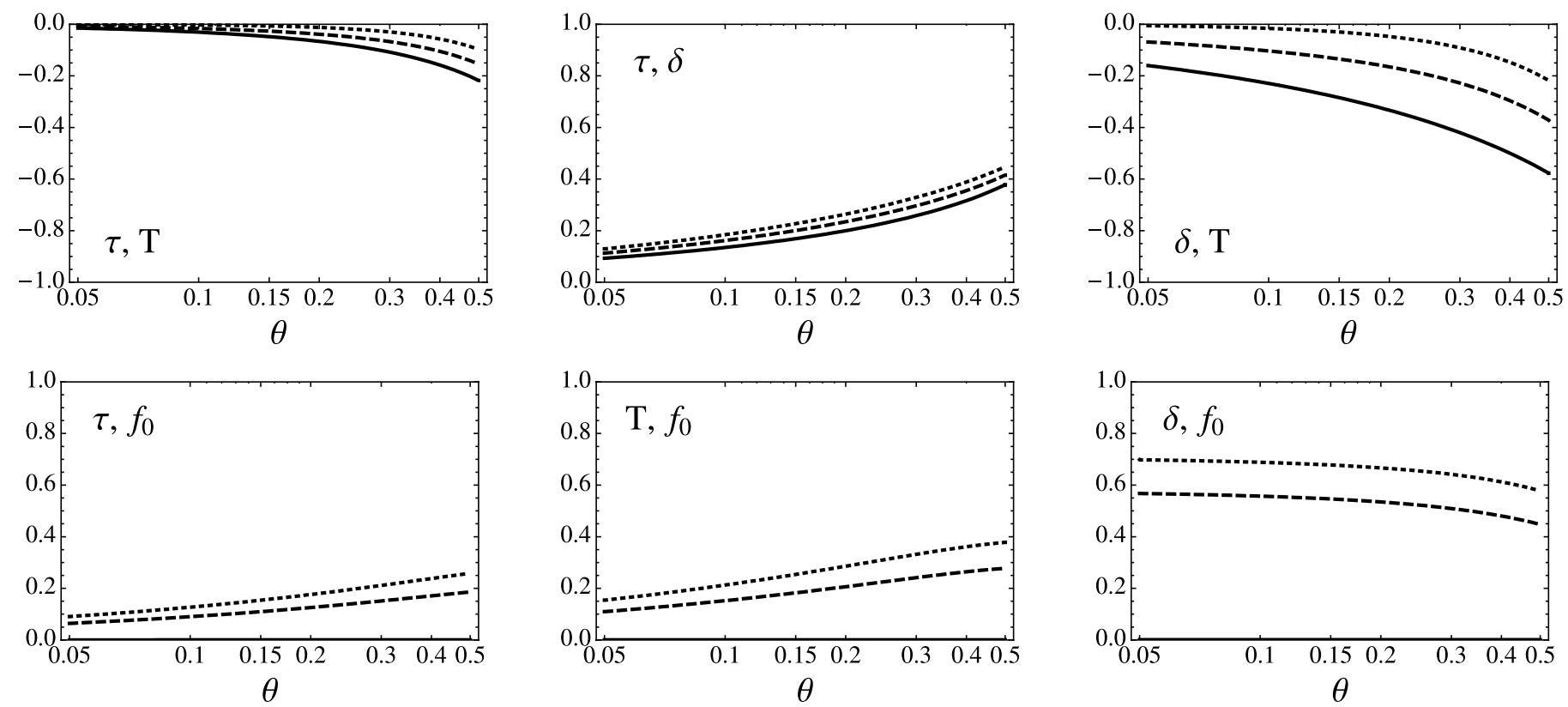

Fig. 5.-Correlations of the piecewise linear model parameters, as a function of $\theta \equiv \tau / T$ for different choices of $\eta$. We show results for $\eta=0$ (solid lines), $\eta=0.5$ (dashed lines), and $\eta=1$ (dotted lines).

where $a=(\beta-1) \theta /([6-\theta(5-\beta)][2-\theta(1-\beta)])^{1 / 2}, b=((\beta+$ 1) $\theta /[6-\theta(5-\beta)])^{1 / 2}, \quad c=(\beta \theta /[6-\theta(5-\beta)])^{1 / 2}, \quad d=(\beta-$ 1) $\theta^{1 / 2} /((\beta+1)[2-\theta(1-\beta)])^{1 / 2}, e=(\beta \theta /[2-\theta(1-\beta)])^{1 / 2}$, and $f=[\beta /(\beta+1)]^{1 / 2}$, and we have defined $\beta \equiv \eta(1-\theta)$ to simplify the resulting expression. For $\theta \rightarrow 0$, all correlations with $f_{0}$ vanish except for the correlation with $\delta$. Because of the fact the correlation between $\delta$ and $f_{0}$ is $\propto \beta^{1 / 2}$, it remains large even for fairly small $\beta$. In the limit of $\eta \rightarrow 0(\beta \rightarrow 0)$, we remove all correlations with $f_{0}$ and have the remaining correlations depending only on the ratio $\theta$,

$$
\begin{aligned}
& \lim _{\eta \rightarrow 0} \operatorname{Corr}(\cdot, \cdot)= \\
& \left(\begin{array}{ccccc}
1 & 0 & 0 & \sqrt{\frac{\theta}{6-5 \theta}} & 0 \\
0 & 1 & a & -\sqrt{\frac{\theta}{2-\theta}} & 0 \\
0 & a & 1 & 1 & 0 \\
0 & \sqrt{\frac{\theta}{6-5 \theta}} & -\sqrt{\frac{\theta}{2-\theta}} & 0 & 1
\end{array}\right),
\end{aligned}
$$

where $a=-\theta /[(6-5 \theta)(2-\theta)]^{1 / 2}$. Correlations with $f_{0}$ decline with $\eta$ as $\sqrt{\eta}$.

In Figure 5 we have plotted the nonzero correlations as a function of $\theta$ for a few choices of $\eta$. The special case of $\eta \rightarrow 0$ is plotted in Figure 6. In the $\eta \rightarrow 0$ limit, all correlations are small ( $\lesssim 0.3)$ over a large region of the parameter space. Thus, our choice of parameters provides a weakly correlated set for all but grazing transits $(\theta \sim 1 / 2)$, as noted during the numerical analysis of particular systems by Burke et al. (2007) and Bakos et al. (2007). One naturally wonders whether a different choice of parameters would give even smaller (or even zero) correlations. In $\S 6$ we present parameter sets that are essentially uncorrelated and have other desirable properties for numerical parameter estimation algorithms.
The analytic formalism given in this section and, more specifically, the simple analytic covariance matrices in equations (20) and (21) provide a toolbox with which to evaluate the statistical merits of any parameter set that can be written in terms of our parameters. In $\S 5$ this technique is defined and applied to produce analytic formulae for variances, covariances, and uncertainties in several interesting parameters.

\section{ACCURACY OF THE COVARIANCE EXPRESSIONS}

Before investigating other parameter sets, it is necessary to examine the validity of equations (20), (21), (24), and (25) when compared to similar quantities derived from more realistic transit light-curve models. The utility of the covariance matrix in equation (20) depends on the accuracy of the integral approximation of equation (17) and on the fidelity with which the parameter dependences of the piecewise linear model mimic the dependences

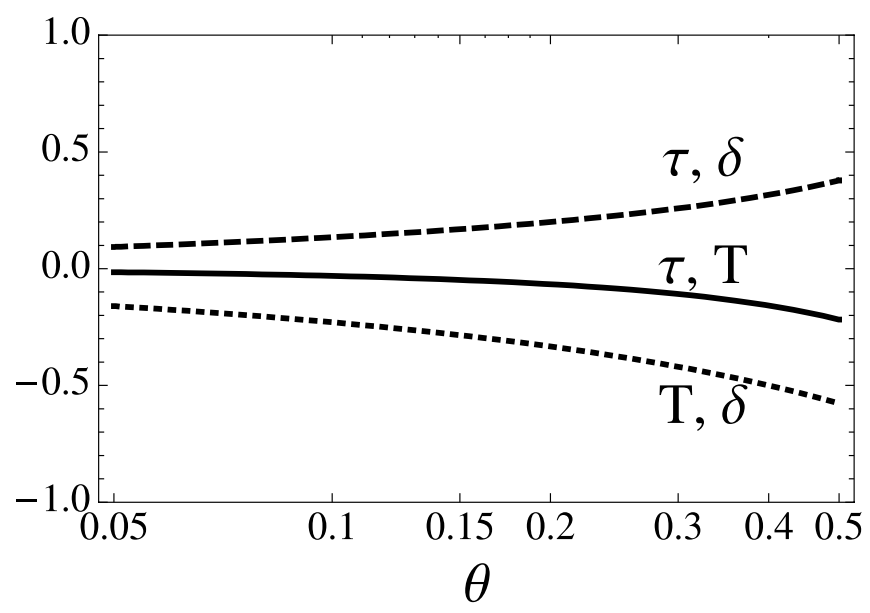

FIG. 6.-Correlations of the piecewise linear model parameters, as a function of $\theta \equiv \tau / T$, for the case $\eta \rightarrow 0$ (errorless knowledge of the out-of-transit flux). The solid line denotes $\operatorname{Corr}(\tau, T)$, the dashed line denotes $\operatorname{Corr}(\tau, \delta)$, and the dotted line denotes $\operatorname{Corr}(T, \delta)$. 
of the exact uniform-source model. In this section we investigate these two issues.

\subsection{Finite Cadence Correction}

The case of a finite observing cadence, rather than continuous sampling, can be analyzed by evaluating the exact sums of equation (16). Generally, given a sampling rate $\Gamma$, we expect the integral approximation in equation (17) to be valid to order $(\Gamma \tau)^{-1}$. In the $\eta \rightarrow 0$ limit we may evaluate the exact sums, under the assumption of a uniform sampling rate, with data points occurring exactly at the start and end of the ingress (and egress) phases as well as at some intermediate times. This directly summed covariance, $\operatorname{Cov}_{\text {sum }}$, is related to the integral approximation covariance equation (21) as

$$
\begin{aligned}
\operatorname{Cov}_{\text {sum }}(\cdot, \cdot)= & \operatorname{Cov}(\cdot, \cdot)+6\left(\frac{T}{Q}\right)^{2} \\
& \times \frac{\theta}{1-\epsilon^{2}}\left(\begin{array}{cccc}
0 & 0 & 0 & 0 \\
0 & \epsilon^{2} & \epsilon & 0 \\
0 & \epsilon & \epsilon^{2} & 0 \\
0 & 0 & 0 & 0
\end{array}\right),
\end{aligned}
$$

where $\epsilon=(\Gamma \tau)^{-1}$.

The quantity $\Gamma \tau$ is approximately the number of data points obtained during ingress or egress. It is evident from equation (26) that for this sampling scheme only the variances of $T$ and $\tau$ along with their covariance are corrected. The corrections to the variances and covariance are $O\left(\epsilon^{2}\right)$ and $O(\epsilon)$, respectively.

\subsection{Comparison with Covariances of the Exact Uniform-Source Model}

We tested the accuracy of the covariance matrix based on the piecewise linear model by (1) performing a numerical Fisher analysis of the exact uniform-source model and also (2) applying a Markov chain Monte Carlo (MCMC) analysis of simulated data based on the exact uniform-source model. In both analyses, orbits are assumed to be circular. For the first task, we evaluated the analytic parameter derivatives of equation (1), which are too cumbersome to be worth reproducing here, and numerically integrated equation (17) to generate covariance matrices over a wide range of parameter choices. Figure 2, in $\S 2$, shows the parameter derivatives for the exact model, as well as the piecewise linear model and some limb-darkened light curves. For the second task, idealized data was generated by adding Gaussian noise with standard deviation $\sigma / f_{0}=5 \times 10^{-4}$ to equation (1) sampled at $\Gamma=100$ (in units of the characteristic timescale $\tau_{0}$, eq. [7]). With this sampling rate, approximately 50 samples occur during the ingress and egress phases. Approximately $10^{4}$ links per parameter were generated with a Gibbs sampler and a Metropolis-Hasting jump acceptance criterion. The jump success fraction (the fraction of jumps in parameter space that are actually executed) was approximately $25 \%$ for all parameters. The effective length, defined as the ratio of the number of links to the correlation length (see the end of $\S 6$ for the exact definition), was roughly 1000-2000 for the piecewise linear model parameter set. More details on the MCMC algorithm are given by Tegmark et al. (2004) and Ford (2005). Standard errors were determined by computing the standard deviation of the resulting distribution for each parameter. The Fisher information analysis should mirror the MCMC results, as long as the log-likelihood function is well approximated as quadratic near the mean (Gould 2003).

The numerical Fisher analysis was performed for $\eta=0$ and $0.05 \leq \theta \lesssim 1 / 2$. In practice this was done by choosing $r=0.05$

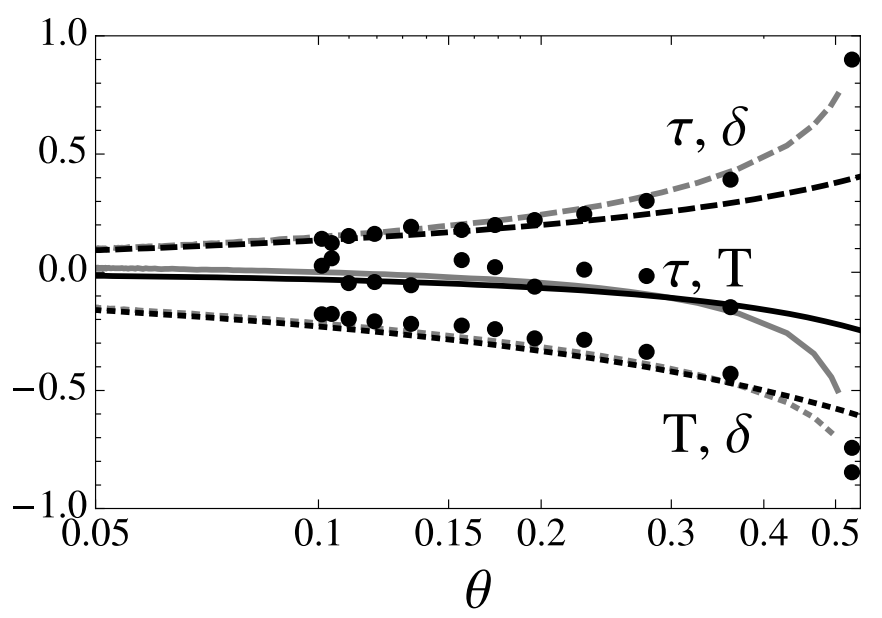

FIG. 7.- Comparison of the nonzero correlation matrix elements for the exact light-curve model and the piecewise linear model, as a function of $\theta \equiv \tau / T$, for $\eta \rightarrow 0$. Black lines show correlations for the piecewise linear model. Gray lines show correlations for the exact uniform-source model. Filled circles show correlations based on an MCMC analysis of simulated data with Gaussian noise $(r=0.1)$.

and varying $b$ across the full range of impact parameters. (The numerical analysis confirmed that the suitably normalized covariances vary only as a function of $\theta \equiv \tau / T$, with the exception of a slight $\delta$-dependent positive offset in $\sigma_{\delta}$ that goes to zero as $\delta$ goes to zero.) The MCMC analysis for $\eta=0$ was accomplished by fixing the out-of-transit flux, $f_{0}=1$, and varying the remaining parameters. We chose $r=0.1$ for the MCMC analysis. Figure 7 shows all of the nonzero numerical correlation matrix elements, as a function of $\theta$. The MCMC results, plotted as filled circles, closely follow the curves resulting from the numerical Fisher analysis. Figure 8 shows the nonzero numerical covariance matrix elements, also for the case $\eta=0$.

The correlations of the piecewise linear model match the correlations of the exact model reasonably well, with the most significant deviations occurring only in the grazing limit, $\theta \sim 1 / 2$. We have also confirmed that a similar level of agreement is obtained for nonzero $\eta$, although for brevity those results are not shown here. We concluded from these tests that the errors in the analytic estimates of the uncertainties are generally small enough for the analytic error estimates derived from the piecewise linear model to be useful.

\subsection{The Effects of Limb Darkening}

The piecewise linear function of equation (11) was constructed as a model of a transit across a stellar disk of uniform brightness, with applications to far-red and infrared photometry in mind. At shorter wavelengths, the limb darkening of the star is important. How useful are the previously derived results for this case, if at all? We used the limb-darkened light-curve models given by Mandel \& Agol (2002) to answer this question.

To simplify the analysis we adopted a "linear" limb-darkening law, in which the surface brightness profile of the star is

$$
\frac{I(z)}{I_{0}}=1-u\left(1-\sqrt{1-z^{2}}\right),
$$

where $u$ is the linear limb-darkening parameter. Claret (2000) finds values of $u$ ranging from 0.5 to 1.2 in $U B V R$ for a range of main-sequence stars. Longer wavelength bands correspond to a smaller $u$ for the same surface gravity and effective temperature. Solar values are $u \approx 0.5$ in the Johnson $R$ band and 0.2 in the 

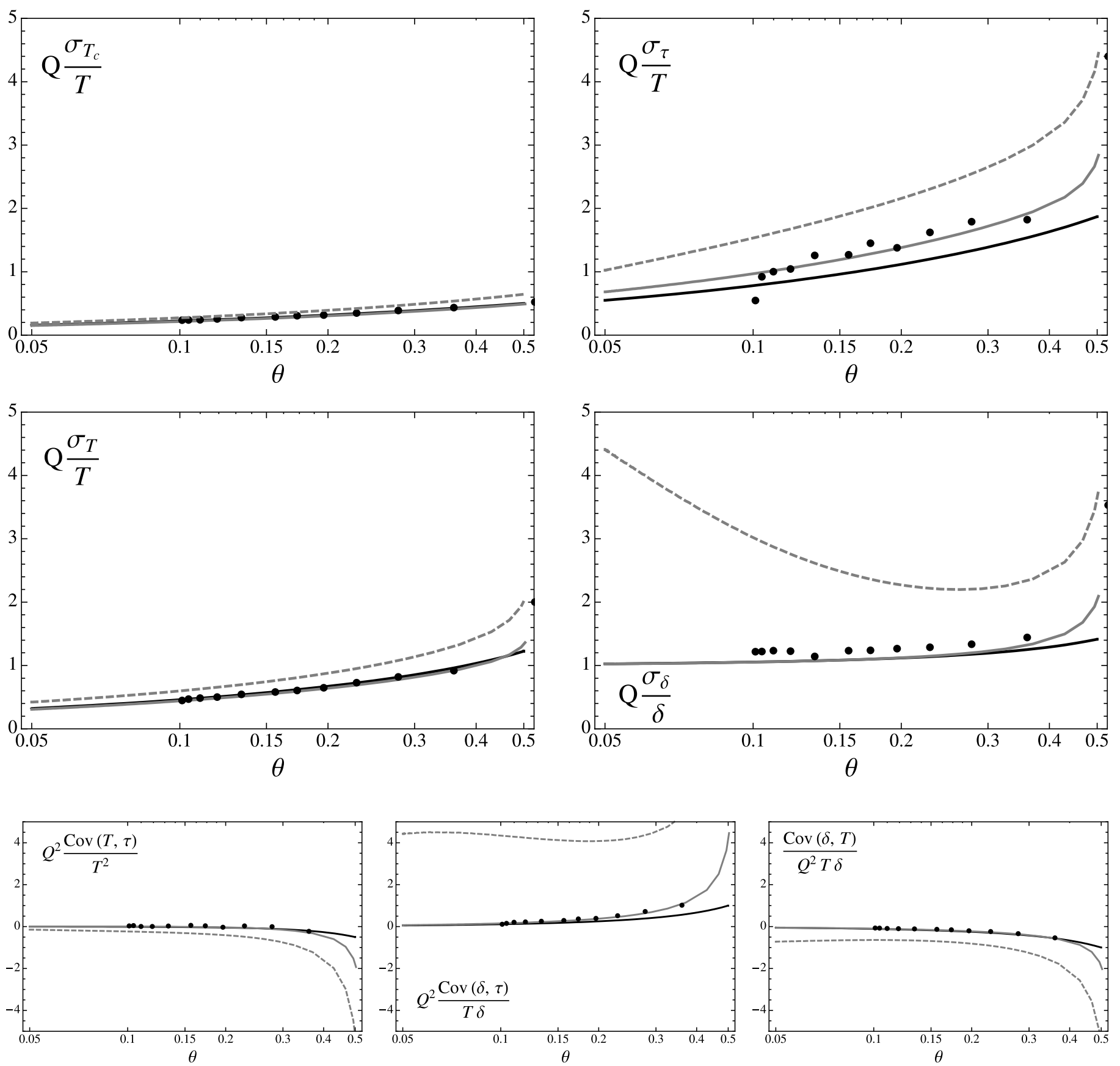

FIG. 8.-Comparison of the covariance matrix elements for the exact uniform-source model, linear limb-darkened model, and the piecewise linear model, as a function

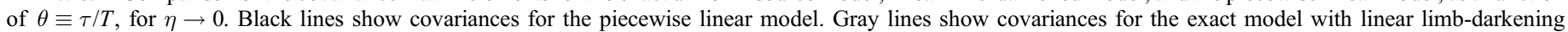
coefficient $u=0$ (solid lines) and 0.5 (dashed lines). Filled circles show covariances as determined by a MCMC analysis of simulated data with Gaussian noise $(u=0$ and $r=0.1$ ). The dimensionless number $Q \equiv(\Gamma T)^{1 / 2} \delta / \sigma$ (see eq. [19]) is approximately the signal-to-noise ratio of the transit.

$K$ band. Figure 2 of $\S 2$ shows the time dependence of the parameter derivatives of a linear limb-darkened light curve, for the two cases $u=0.2$ and 0.5 , to allow for comparison with the corresponding dependences of the piecewise linear model and the exact model with no limb darkening.

From the differences apparent in this plot, one would expect increased correlations (larger than our analytic formulae would predict) between the transit depth and the two timescales $\tau$ and $T$. This is borne out by our numerical calculations of the covariance matrix elements, which are plotted in Figures 8 and 9. The analytic formulae underpredict the variances in $\delta$ and $\tau$ by a factor of a few, and they also severely underpredict the correlation between those parameters.
It is possible to improve the agreement with the analytic formulae by associating $\delta$ with the minimum of the transit light curve, rather than the square of the radius ratio. Specifically, one replaces the definition of equation (12) with the new definition

$$
\delta=f_{0} r^{2} \frac{9-8\left(\sqrt{1-b^{2}}-1\right) u}{9-8 u} .
$$

For the previously derived formulae to be valid, we must adopt a value for $u$ based on other information about the parent star (its spectral energy distribution and spectral lines, luminosity, etc.) rather than determining $u$ from the photometric data. Figure 10 shows the correlations resulting from this new association, for the 

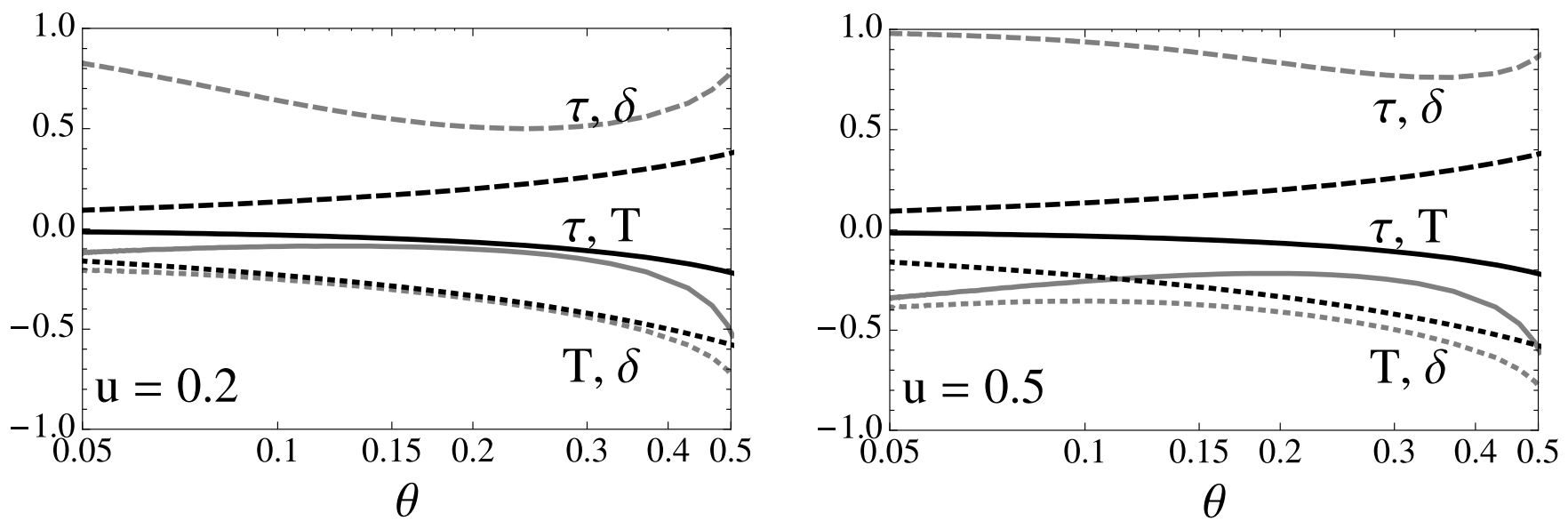

FIG. 9.-Comparison of the analytic correlations (black lines; eq. [24]) and numerically calculated correlation matrix elements for a linear limb-darkened light curve (gray lines), as a function of $\theta \equiv \tau / T$, for $\eta \rightarrow 0$. Line styles follow the conventions of Fig. 7.

case $u=0.5$. Figure 11 shows the improvement with this new association for the variance in $\delta$ and the covariance between $\delta$ and $\tau$, for the case $u=0.5$. While this new association improves on the agreement with the analytic covariances (particularly at low normalized impact parameters), a disadvantage is that we no longer have a closed-form mapping from $\{\delta, T, \tau\}$ back to the more physical parameters $\left\{r, b, \tau_{0}\right\}$.

It should be noted that there is evidence that linear limb darkening may not adequately fit high-quality transit light curves relative to higher order models (Brown et al. 2001; Southworth 2008). A more complete analysis with arbitrary source surface brightness would minimally include quadratic limb darkening, but is outside the scope of this discussion. Pál (2008) completes a complementary analysis to this one of uncertainties in the quadratic limb-darkening parameters themselves.

\section{ERRORS IN DERIVED QUANTITIES OF INTEREST IN THE ABSENCE OF LIMB DARKENING}

The parameters $\left\{t_{c}, \tau, T, \delta, f_{0}\right\}$ are preferred mainly because they lead to simple analytic formulae for their uncertainties and covariances. The values of these parameters are also occasionally of direct interest. In particular, when planning observations, it is useful to know the transit duration, depth, and the predicted mid-

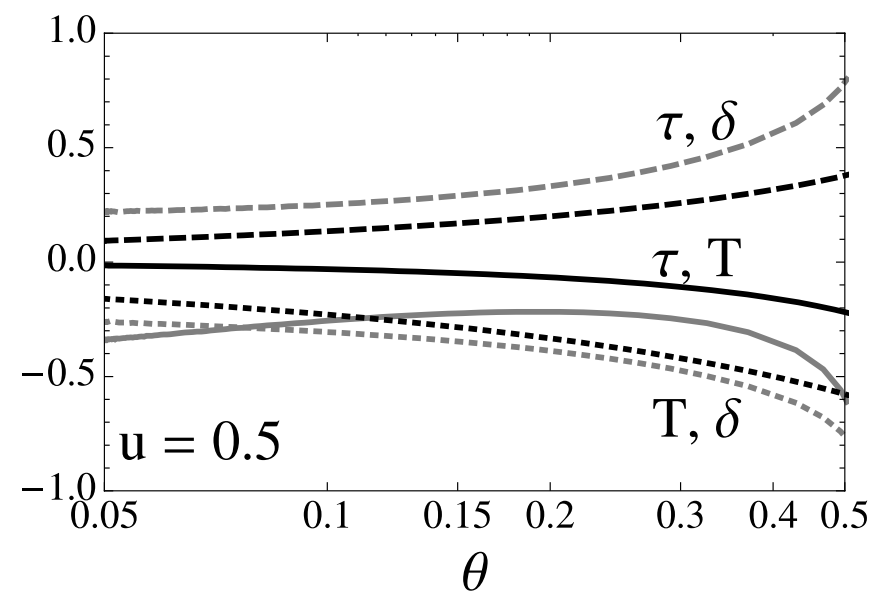

FIG. 10.-Comparison of correlation matrix elements for the piecewise linear model (black lines) and a linear limb-darkened light curve $(u=0.5$; gray lines $)$, as a function of $\theta \equiv \tau / T$. Here, the $\delta$-parameter has been redefined as the minimum of the limb-darkened light curve, as approximated by eq. (28). Line styles follow the conventions of Fig. 7. transit time. Of more direct scientific interest are the values of the "physical" parameters, such as the planetary and stellar radii, the orbital inclination, and the mean density of the star. Those latter parameters also offer clearer a priori expectations, such as a uniform distribution in $\cos i$.

For affine parameter transformations $\mathbf{p} \mapsto \mathbf{p}^{\prime}$, we may transform the covariance matrix $\mathbf{C}$ via the Jacobian $\mathbf{J}=\partial \mathbf{p}^{\prime} / \partial \mathbf{p}$ as

$$
\mathbf{C}^{\prime}=\mathbf{J}^{T} \mathbf{C} \mathbf{J} \text {. }
$$

Using equations (12)-(14) we may calculate the Jacobian

$$
\begin{aligned}
& \frac{\partial t_{c}, b^{2}, \tau_{0}^{2}, r, f_{0}}{\partial t_{c}, \tau, T, \delta, f_{0}}= \\
& \left(\begin{array}{ccccc}
1 & 0 & 0 & 0 & 0 \\
0 & \frac{r T}{\tau^{2}} & \frac{T}{4 r} & 0 & 0 \\
0 & -\frac{r}{\tau} & \frac{\tau}{4 r} & 0 & 0 \\
0 & -\frac{T}{2 f_{0} r \tau} & -\frac{T \tau}{8 f_{0} r^{3}} & \frac{1}{2 f_{0} r} & 0 \\
0 & \frac{p T}{2 f_{0} \tau} & \frac{T \tau}{8 f_{0} r} & -\frac{r}{2 f_{0}} & 1
\end{array}\right)
\end{aligned}
$$

between the parameters of the piecewise linear model and the more physical parameter set when limb darkening is negligible. Using this Jacobian, the transformed covariance matrix is

$$
\begin{aligned}
& \operatorname{Cov}^{\prime}\left(\left\{b^{2}, \tau_{0}^{2}, r, f_{0}\right\},\left\{b^{2}, \tau_{0}^{2}, r, f_{0}\right\}\right) \\
& =\frac{1}{Q^{2}}\left(\begin{array}{cccc}
a r^{2} & b T^{2} & d r^{2} & 0 \\
b T^{2} & c \theta T^{4} & e \theta T^{2} & 0 \\
d r^{2} & e \theta T^{2} & f r^{2} & 0 \\
0 & 0 & 0 & 0
\end{array}\right) \\
& +\frac{\eta}{Q^{2}}\left(\begin{array}{cccc}
\frac{g^{2}}{4 \theta^{2}} r^{2} & \frac{1}{16} i T^{2} & \frac{g}{4 \theta} r^{2} & \frac{r^{3} g}{2 \theta} f_{0} \\
\frac{1}{16} i T^{2} & \frac{\theta^{2} h^{2}}{64 r^{2}} T^{4} & \frac{1}{16} \theta h T^{2} & \frac{1}{8} r \theta h f_{0} T^{2} \\
\frac{g}{4 \theta} r^{2} & \frac{1}{16} \theta h T^{2} & \frac{1}{4} r^{2} & \frac{1}{2} r^{3} f_{0} \\
\frac{r^{3} g}{2 \theta} f_{0} & \frac{1}{8} r \theta h f_{0} T^{2} & \frac{1}{2} r^{3} f_{0} & r^{4} f_{0}^{2}
\end{array}\right),
\end{aligned}
$$



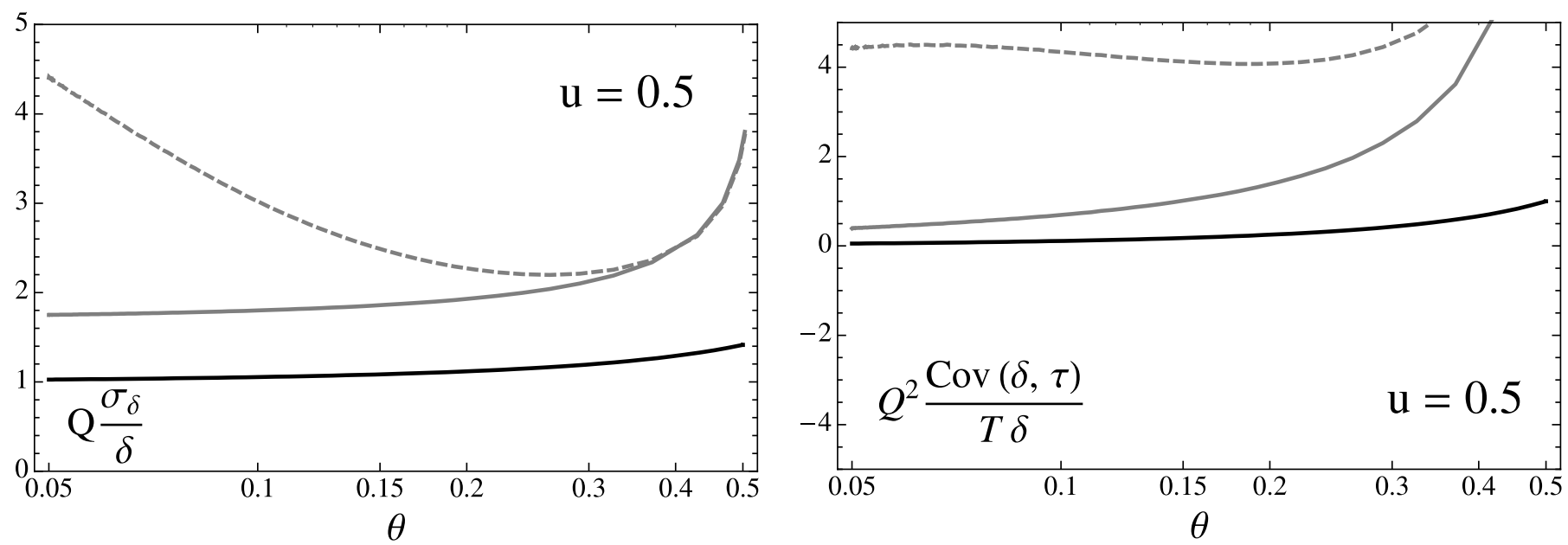

FIG. 11. - Comparison of select covariance matrix elements for the piecewise linear model (black lines) and a linear limb-darkened light curve ( $u=0.5 ;$ gray lines), as a function of $\theta \equiv \tau / T$. The $\delta$-parameter has been redefined as the minimum of the limb-darkened light curve, as approximated by eq. (28), in the solid gray line. The dashed gray line uses the initial $\delta$-association, as defined in eq. (8). Line styles follow the conventions of Fig. 8.

where $a=(24-\theta[4(\theta-3) \theta+23]) /\left[4(1-\theta) \theta^{3}\right], \quad b=(24-$ $\theta[23-4(\theta-2) \theta]) /[16(1-\theta) \theta], c=(24-\theta[4(\theta-1) \theta+23]) /$ $\left[64 r^{2}(1-\theta)\right], d=(2 \theta+1) /[4 \theta(1-\theta)], e=(1-2 \theta) /[16(1-$ $\theta)], f=1 /[4(1-\theta)], g=1-2 \theta, h=1+2 \theta$, and $i=1-4 \theta^{2}$, and where we have ignored the unmodified covariance elements involving $t_{c}$ and have kept only the leading-order terms in $r$ in the $\eta$-dependent matrix.

The standard errors for other functions of the parameters, $f\left(\left\{p_{i}\right\}\right)$, can be found via error propagation, just as in equation (29),

$$
\operatorname{Var}\left[f\left(\left\{p_{i}\right\}\right)\right]=\sum_{i} \sum_{j} \operatorname{Cov}\left(p_{i}, p_{j}\right) \frac{\partial f}{\partial p_{i}} \frac{\partial f}{\partial p_{j}} .
$$

The results for several interesting and useful functions, such as the mean densities of the star and planet, are given in Table 2. For brevity, the results are given in terms of the matrix elements of equation (31). Simplified expressions for covariance matrix elements in the limit of $\eta \rightarrow 0$, small $\theta$ (plentiful out-of-transit data), and negligible limb darkening are given in Table 3.

\section{OPTIMIZING PARAMETER SETS FOR FITTING DATA WITH SMALL LIMB DARKENING}

The parameter set $\left\{t_{c}, \tau, T, \delta, f_{0}\right\}$ has the virtues of simplicity and weak correlation over most of the physical parameter space. However, when performing numerical analyses of actual data, the virtue of simplicity may not be as important as the virtue of low correlation, which usually leads to faster and more robust convergence. To take one example, lower correlations among the parameters result in reduced correlation lengths for MCMCs and faster convergence to the desired a posteriori probability distributions and can obviate the need for numerical principal component analysis (Tegmark et al. 2004). In Figure 12 we compare the degree of correlations for various parameter sets that have been used in the literature on transit photometry. Of note is

TABLE 2

Transit Quantities and Associated Variances in Terms of the Matrix Elements from Equation (31)

\begin{tabular}{|c|c|c|}
\hline Quantity & Variance (Standard Error Squared) & Notes \\
\hline$R_{p}=r R_{\star} \ldots \ldots \ldots \ldots \ldots$ & $R_{p}^{2}\left[\operatorname{Var}(r) / r^{2}+\left(\log M_{\star} / M_{\odot}\right)^{2} \operatorname{Var}^{*}(x)\right]$ & 1 \\
\hline 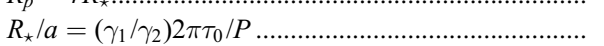 & $(1 / 4)\left(R_{\star} / a\right)^{2} \operatorname{Var}\left(\tau_{0}^{2}\right) / \tau_{0}^{4}$ & \\
\hline 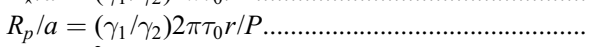 & $\left(R_{p} / a\right)^{2}\left[(1 / 4) \operatorname{Var}\left(\tau_{0}^{2}\right) / \tau_{0}^{4}+\operatorname{Var}(r) / r^{2}\right]$ & \\
\hline$|b|=\left(\gamma_{2}^{2} / \gamma_{1}\right)\left|a \cos i / R_{\star}\right| \ldots \ldots \ldots \ldots \ldots \ldots \ldots \ldots \ldots \ldots \ldots \ldots \ldots$ & $(1 / 4) \operatorname{Var}\left(b^{2}\right) / b^{2}$ & \\
\hline 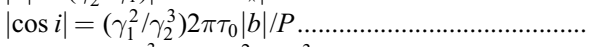 & $(1 / 4) \cos ^{2} i\left[\operatorname{Var}\left(b^{2}\right) / b^{4}+\operatorname{Cov}\left(\tau_{0}^{2}, b^{2}\right) / \tau_{0}^{2} b^{2}+\operatorname{Var}\left(\tau_{0}^{2}\right) / \tau_{0}^{4}\right]$ & \\
\hline$\rho_{\star}=\left(\gamma_{2} / \gamma_{1}\right)^{3}\left(3 / 8 G \pi^{2}\right) P / \tau_{0}^{3} \ldots \ldots \ldots \ldots \ldots$ & $(9 / 4) \rho_{\star}^{2} \operatorname{Var}\left(\tau_{0}^{2}\right) / \tau_{0}^{4}$ & \\
\hline$\rho_{p}=\gamma_{2}\left(K_{\star} \rho_{\star} / r^{3} \sin i\right)\left(P / 2 \pi G M_{\star}\right)^{1 / 3} \ldots \ldots \ldots \ldots \ldots \ldots \ldots \ldots \ldots \ldots \ldots \ldots \ldots$ & $\begin{array}{l}\rho_{p}^{2}\left[(9 / 4) \operatorname{Var}\left(\tau_{0}^{2}\right) / \tau_{0}^{4}+9 \operatorname{Var}(r) / r^{2}+(9 / 2) \operatorname{Cov}\left(r, \tau_{0}^{2}\right) / r \tau_{0}^{2}+\right. \\
\quad(1 / 4)(\cos i / b)^{4} \operatorname{Var}\left(b^{2}\right)-(3 / 4)(\cos i / b)^{2} \operatorname{Cov}\left(b^{2}, \tau_{0}^{2}\right) / \tau_{0}^{2}- \\
\left.\quad(3 / 2)(\cos i / b)^{2} \operatorname{Cov}\left(b^{2}, r\right) / r+\operatorname{Var}^{*}\left(K_{\star}\right) / K_{\star}^{2}\right]\end{array}$ & 2 \\
\hline  & $g_{\star}^{2}\left[(9 / 4) \operatorname{Var}\left(\tau_{0}^{2}\right) / \tau_{0}^{4}+\left(\log M_{\star} / M_{\odot}\right)^{2} \operatorname{Var}^{*}(x)\right]$ & 1 \\
\hline 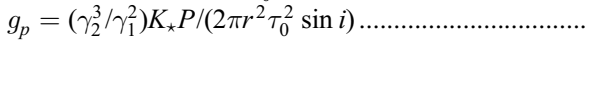 & $\begin{array}{l}g_{p}^{2}\left[\operatorname{Var}\left(\tau_{0}^{2}\right) / \tau_{0}^{4}+4 \operatorname{Var}(r) / r^{2}+2 \operatorname{Cov}\left(r, \tau_{0}^{2}\right) / r \tau_{0}^{2}+\right. \\
\quad(1 / 4)(\cos i / b)^{4} \operatorname{Var}\left(b^{2}\right)-(1 / 2)(\cos i / b)^{2} \operatorname{Cov}\left(b^{2}, \tau_{0}^{2}\right) / \tau_{0}^{2}- \\
\left.\quad(\cos i / b)^{2} \operatorname{Cov}\left(b^{2}, r\right) / r+\operatorname{Var}^{*}\left(K_{\star}\right) / K_{\star}^{2}\right]\end{array}$ & 2 \\
\hline
\end{tabular}

Notes.-We have assumed that both the orbital period, $P$, and stellar mass, $M_{\star}$, are known exactly. We have defined the noncircular orbit parameters $\gamma_{1} \equiv 1+e \sin \omega$ and $\gamma_{2} \equiv\left(1-e^{2}\right)^{1 / 2}$, where $e$ is the eccentricity and $\omega$ is the argument of pericenter (see $\S 2$ for a discussion of eccentric orbits). Quantities with asterisks are not determined by the transit model and must be provided from additional information. The term $K_{\star}$ is the semiamplitude of the source radial velocity. Terms have been arranged in order of relative importance with the largest in absolute magnitude coming first. Refer to Table 3 for matrix elements of eq. (31) for the case in which the planet is small, the out-of-transit flux is known precisely, and limb darkening is negligible. Notes from the last column: (1) A mass-radius relation $R_{\star} \propto\left(M_{\star} / M_{\odot}\right)^{x}$ is assumed. (2) We have assumed $i \gtrsim 80^{\circ}$ in simplifying the inclination dependence in the variance. 
TABLE 3

Covariance Matrix Elements from Equation (31) In the Limit $\eta \rightarrow 0$ and Small $\theta$ for Use in Table 2

\begin{tabular}{|c|c|}
\hline Element & Approximate Value \\
\hline$Q^{2} \operatorname{Var}(r) / r^{2}$ & 14 \\
\hline$Q^{2} \operatorname{Var}\left(b^{2}\right) / b^{4}$. & $6 r^{2} / \theta^{3} b^{4}$ \\
\hline$Q^{2} \operatorname{Var}\left(\tau_{0}^{2}\right) / \tau_{0}^{4}$. & $3 / 2 \theta$ \\
\hline$Q^{2} \operatorname{Cov}\left(b^{2}, \tau_{0}^{2}\right) / b^{2} \tau_{0}^{2}$. & $6 r / \theta^{2} b^{2}$ \\
\hline  & $r / 4 \theta b^{2}$ \\
\hline 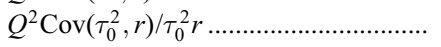 & $1 / 16$ \\
\hline
\end{tabular}

Note.-These approximations are valid in the case in which the planet is small, the out-of-transit flux is known precisely, and limb darkening is negligible.

the high degree of correlations among the "physical" parameter set $\left\{R_{\star} / a, R_{p} / a, b\right\}$, which is a poor choice from the point of view of computational speed.

Nevertheless, one advantage of casting the model in terms of physical parameters is that the a priori expectations for those parameters are more easily expressed, such as a uniform distribution in $b$. The determinant of the Jacobian given by equation (29), $|\mathbf{J}|$, is also useful in translating a priori probability distributions from one parameter set to the other (see Burke et al. [2007] or Ford [2006] for an example of how this is done in
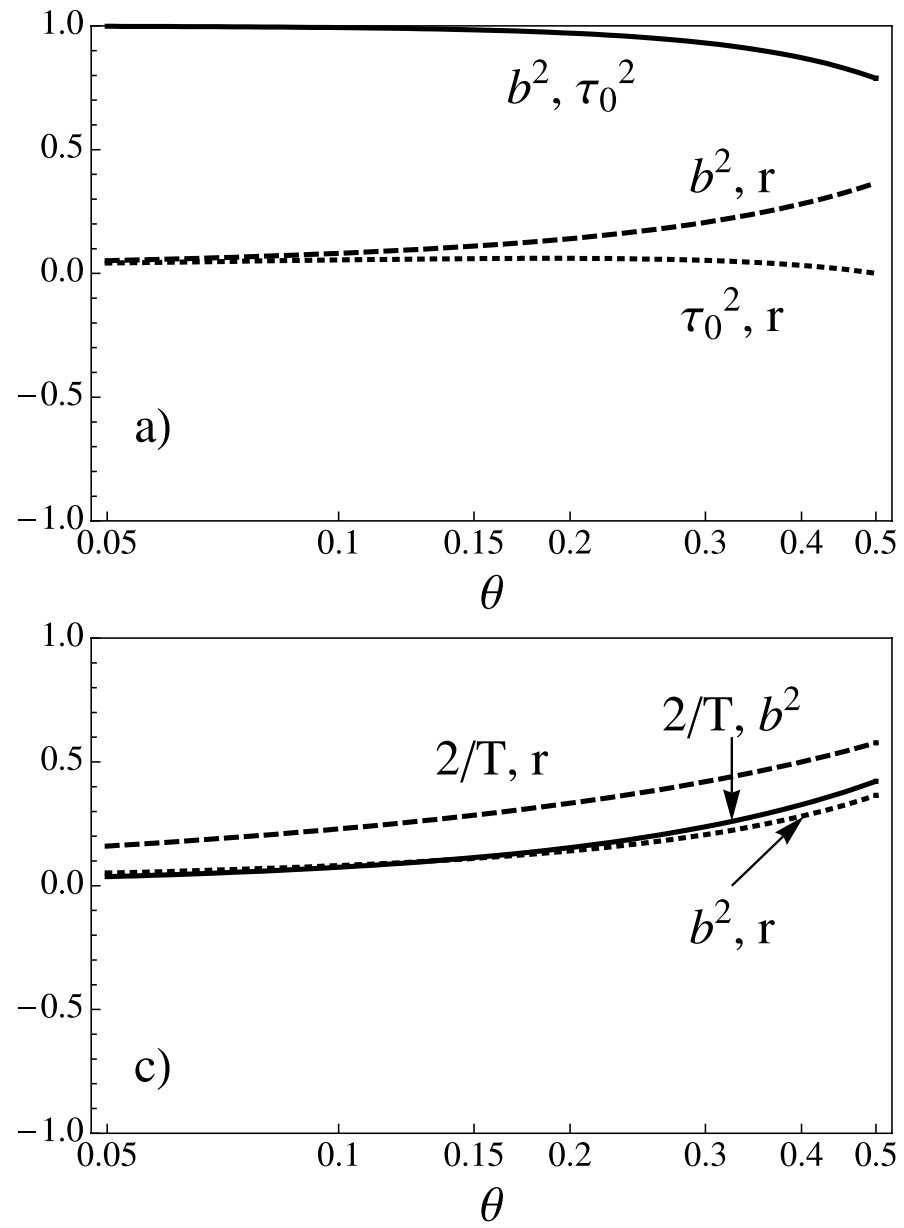

practice). For the case of the parameter set $\left\{t_{c}, \tau, T, \delta, f_{0}\right\}$, we may use the Jacobian, equation (30), to convert a priori probability distributions via

$$
\begin{aligned}
& p\left(t_{c}, \tau, T, \delta, f_{0}\right) d t_{c} d \tau d T d \delta d f_{0} \\
& =p\left(t_{c}, b^{2}, \tau_{0}^{2}, r, f_{0}\right) \frac{1}{4 r \theta f_{0}} d t_{c} d b^{2} d \tau_{0}^{2} d r d f_{0} \\
& =p\left(t_{c}, b, \tau_{0}, r, f_{0}\right) \frac{1}{4 r \theta f_{0}} \frac{1}{4 b \tau_{0}} d t_{c} d b d \tau_{0} d r d f_{0} \\
& =p\left(t_{c}, b, \tau_{0}, r, f_{0}\right)\left(\frac{1-b^{2}}{16 b r^{2} \tau_{0} f_{0}}\right) d t_{c} d b d \tau_{0} d r d f_{0},
\end{aligned}
$$

where we have remeasured the phase-space volume via the determinant,

$$
\left\|\frac{\partial\left\{t_{c}, b^{2}, \tau_{0}^{2}, r, f_{0}\right\}}{\partial\left\{t_{c}, \tau, T, \delta, f_{0}\right\}}\right\|=\frac{1}{4 r \theta f_{0}} .
$$

One may use this expression to enforce a uniform prior in $b$, for example, by weighting the likelihood function as shown in equation (33). However, there is a practical difficulty due to the singularity at $b=0$. One way to understand the singularity is
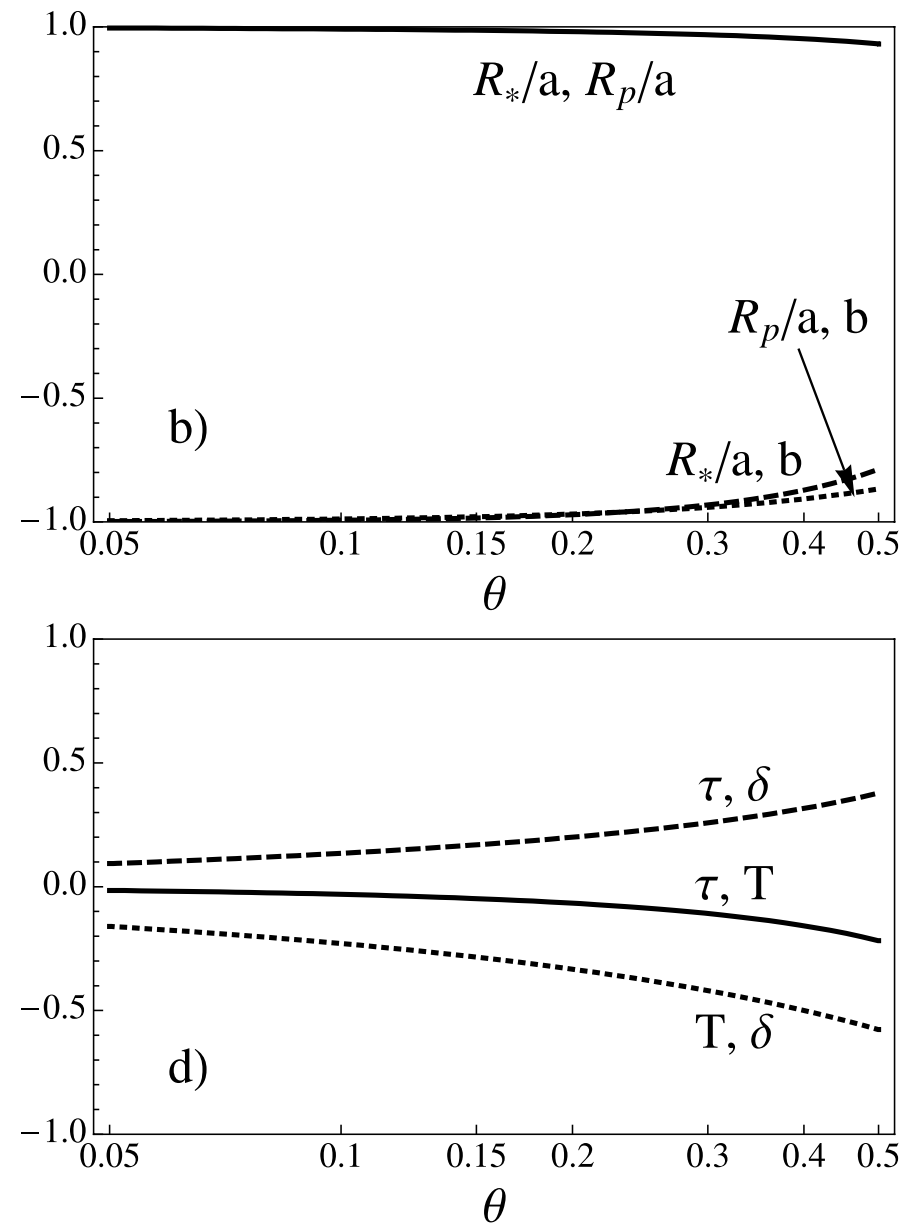

FIG. 12.-Comparison of correlations for various parameter sets that have been used in the literature. The correlations were derived from the piecewise linear model

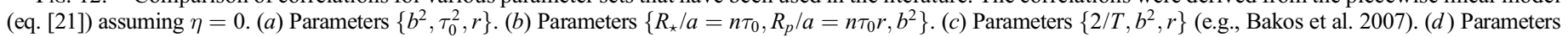
$\{T, \tau, \delta\}$, the set introduced in this paper. 


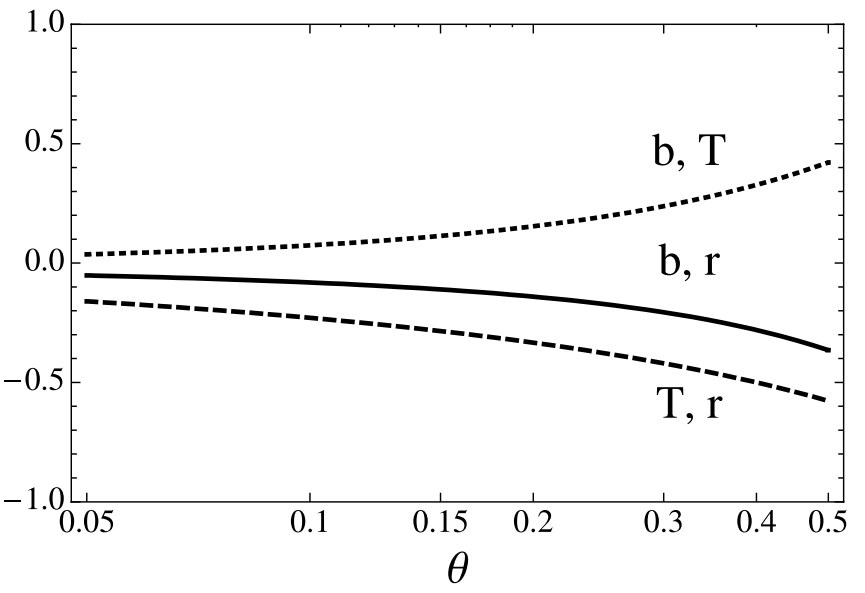

FIG. 13.-Correlations for the parameter set $\{b, T, r\}$. The correlations were derived from the piecewise linear model (eq. [21]) assuming $\eta=0$.

to note that uniform distributions in $\tau$ and $T$ lead to a nearly uniform distribution in $\theta=\tau / T$, which highly disfavors $b=0$; in order to enforce a uniform distribution in $b$, the prior must diverge at low $b$. Figure 3 graphically captures the steep variation for small $b$ with $\theta$. Consider, instead, the parameter set $\left\{t_{c}, b, T, r \equiv\left(\delta / f_{0}\right)^{1 / 2}, f_{0}\right\}$, where from equation $(13), b^{2}=1-$ $r T / \tau$. We may calculate the determinant of the Jacobian (not reproduced here) as

$$
\left\|\frac{\partial\left\{t_{c}, b, T, r, f_{0}\right\}}{\partial\left\{t_{c}, \tau, T, \delta, f_{0}\right\}}\right\|=\frac{\left(1-b^{2}\right)^{2}}{4 b r^{2} f_{0} T} .
$$

Combining this result with equation (33),

$$
\begin{aligned}
& p\left(t_{c}, b, T, r, f_{0}\right) d t_{c} d b d T d r d f_{0} \\
& =p\left(t_{c}, b, \tau_{0}, r, f_{0}\right) \frac{1}{1-b^{2}} \frac{T}{4 \tau_{0}} d t_{c} d b d \tau_{0} d r d f_{0} \\
& =p\left(t_{c}, b, \tau_{0}, r, f_{0}\right) \frac{1}{2 \sqrt{1-b^{2}}} d t_{c} d b d \tau_{0} d r d f_{0} .
\end{aligned}
$$

The singularity at $b=0$ has been removed with this parameter choice. There is a singularity at $b=1$ instead, which is only relevant for near-grazing transits, and is not as strong of a singularity because of the square root. We confirm that this parameter set also enjoys weak correlations, as shown in Figure 13, and therefore, this set is a reasonable choice for numerical parameter estimation algorithms. The merits of other parameter sets, from the standpoint of correlation and a priori likelihoods, may be weighed in a similar fashion, using the simple analytic covariance matrix of equation (20) and the appropriate transformation Jacobian, in combination with equation (29).

If the issues associated with the transformation of priors are ignored (i.e., if the data are of such quality that the results will depend negligibly on the priors), we can give essentially uncorrelated parameter sets. Consider, for example, the parameter set $\left\{t_{c}, S_{e} \equiv \delta / \tau, T, A \equiv \delta T\right\}$. The new parameter $S_{e}$ is the magnitude of the slope of the light curve during the ingress and egress phases, and the new parameter $A$ is the area of the trapezoid defined by the transit portion of the light curve (i.e., the time integral of the flux decrement). For simplicity we assume $\eta=0$

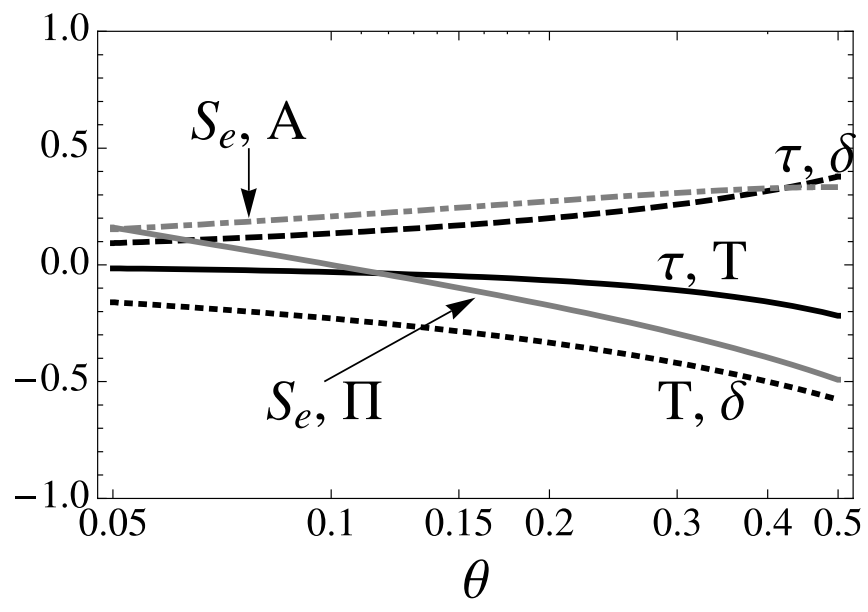

FIG. 14.-Comparison of the correlations among the parameters, for the set $\{\delta, T, \tau\}$ (black lines), the set $\left\{S_{e}, T, A=T \delta\right\}$ (dash-dotted gray line), and the set $\left\{S_{e}, \Pi \equiv T \delta^{\tilde{\theta}}, \delta\right\}$ (solid gray line) for the case $\tilde{\theta}=0.1$. For the latter set, the only nonzero correlation is between $\Pi$ and $S_{e}$, which vanishes at $\theta=0.1$.

and fix $f_{0}=1$. The transformed correlation (eq. [25]) is found via the transformation Jacobian, equation (29), as

$$
\begin{aligned}
& \operatorname{Corr}\left(\left\{t_{c}, S_{e}, T, A\right\},\left\{t_{c}, S_{e}, T, A\right\}\right) \\
& =\left(\begin{array}{cccc}
1 & 0 & 0 & 0 \\
0 & 1 & 0 & 0 \\
0 & 0 & 1 & \sqrt{\frac{\theta(1-\theta)}{(2-\theta)(\theta+1)}} \\
0 & 0 & \sqrt{\frac{\theta(1-\theta)}{(2-\theta)(\theta+1)}} & 1
\end{array}\right) .
\end{aligned}
$$

The determinant of the transformation Jacobian (for use with eq. [33]) is given as

$$
\left\|\frac{\partial\left\{t_{c}, S_{e}, T, A\right\}}{\partial\left\{t_{c}, \tau, T, \delta\right\}}\right\|=\frac{\left(1-b^{2}\right)^{2}}{T} .
$$

With this new parameter set, the only nonzero correlation is between $T$ and $A$, and this correlation is $\lesssim 0.3$ even for grazing transits (see Fig. 14). We have found that these parameters provide a nearly optimal set for data fitting when little is known at the outset about the impact parameter of the transit.

It is possible to do even better when the impact parameter is known at least roughly. Consider the parameter set $\left\{t_{c}, S_{e}, \Pi=\right.$ $\left.T \delta^{\tilde{\theta}}, \delta\right\}$, where $S_{e}$ is the slope of ingress and $\tilde{\theta}$ is a constant (whose chosen value will be discussed momentarily). The new parameter $\Pi$ has no simple physical interpretation. We again assume $\eta=0$ and $f_{0}=1$. The correlation matrix in this case is

$$
\begin{aligned}
& \operatorname{Corr}\left(\left\{t_{c}, S_{e}, \Pi, \delta\right\},\left\{t_{c}, S_{e}, \Pi, \delta\right\}\right)= \\
& \left(\begin{array}{cccc}
1 & 0 & 0 & 0 \\
0 & 1 & 0 & 0 \\
0 & 0 & 1 & (\theta-\tilde{\theta}) \\
0 & 0 & \frac{(\theta-\tilde{\theta})}{\sqrt{(\theta-\tilde{\theta})^{2}+2 \theta(1-\theta)}}
\end{array}\right) .
\end{aligned}
$$


The determinant of the transformation Jacobian is given as

$$
\left\|\frac{\partial\left\{t_{c}, S_{e}, \Pi, \delta\right\}}{\partial\left\{t_{c}, \tau, T, \delta\right\}}\right\|=\frac{\left(1-b^{2}\right)^{2} r^{2 \tilde{\theta}}}{T^{2}} .
$$

With this choice, the only nonzero correlation is between $\Pi$ and $\delta$. If the constant $\tilde{\theta}$ is chosen to be approximately equal to $\theta$, then this sole correlation may be nullified. Thus, if $\theta$ is known even approximately at the outset of data fitting-from visual inspection of a light curve or from the approximation $\theta \approx r$ valid for small planets on nongrazing trajectories - a parameter set with essentially zero correlation is immediately available. As an example, Figure 14 shows the correlation between $\Pi$ and $\delta$ as a function of $\theta$, for the choice $\tilde{\theta}=0.1$, which has a null at $\theta=0.1$ as expected.

The utility of this parameter set is not lost if $\tilde{\theta}$ cannot be confidently specified when used with MCMC parameter estimation codes. At each chain step $i$, the next candidate state can be drawn from the candidate transition probability distribution function generated by the above parameter set with $\tilde{\theta}=\theta_{i-1}$. Thus, the Markov chain will explore the parameter space moving along principal axes at each chain step. In addition, allowing the candidate transition function to vary as the Markov chain explores parameter space may prove useful for low signal-to-noise ratio data sets.

As a concrete example of the effectiveness of uncorrelated parameters, we apply the MCMC algorithm to simulated data. For a given choice of the parameter set, we generate chains with a fixed jump success fraction and calculate the resulting autocorrelations of the Markov chain. For a particular parameter $p$ (with value $p_{i}$ at chain step $i$ ), the autocorrelation $a$ at a given chain step $j$ is defined as

$$
a_{j}=\frac{\left\langle p_{i} p_{i+j}\right\rangle-\left\langle p_{i}\right\rangle^{2}}{\left\langle p_{i}^{2}\right\rangle-\left\langle p_{i}\right\rangle^{2}},
$$

where the averages refer to the averages over the whole chain (Tegmark et al. 2004). The correlation length of the chain is the number of steps $N$ that are required before the autocorrelation drops below 0.5 . The total chain length divided by the correlation length is referred to as the effective length of a chain. The effective chain length is approximately the number of independent samples, which quantifies the degree of convergence of the algorithm. A lower correlation length, for the same total chain length, gives a more accurate final distribution. This autocorrelation analysis was performed for both the "physical" parameter set $\left\{t_{c}, b^{2}, \tau_{0}^{2}, r^{2}\right\}$ as well as the parameter sets $\left\{t_{c}, \tau, T, \delta\right\}$ and $\left\{t_{c}, b, T, r\right\}$, with $\eta=0$ in all cases (i.e., plentiful out-of-transit data). The MCMC was executed as detailed in $\S 4.2$ with a fixed jump rate $\approx 50 \%$ for all parameter chains. (In practice, this was achieved by adjusting the size of the Gaussian random perturbation that was added to each parameter at each trial step.) By choosing either the parameter set $\left\{t_{c}, \tau, T, \delta\right\}$ or $\left\{t_{c}, b, T, r\right\}$, the correlation lengths are reduced by a factor of approximately 150 . By using the minimally correlated parameter set $\left\{t_{c}, S_{e}, T, A\right\}$, the correlation lengths are reduced by an additional factor of $\sim 2$.

To completely eliminate the correlations between parameters, one can diagonalize the symmetric covariance matrix (eq. [37]) and find the linear combinations of parameters that eliminates correlations. This was done by Burke et al. (2007) for the particular case of the transiting planet XO-2b. Analytic expressions for the eigenvectors are available because there are only two entangled parameters. However, these eigenvectors are linear combinations of local parameter values; they do not constitute a global transformation rendering the covariance diagonal. Thus, this procedure is useful for numerical analysis of a particular system, although not for analytic insights.

\section{SUMMARY}

We have presented formulae for uncertainties and covariances for a collection of parameters describing the light curve of an exoplanet transiting a star with uniform brightness. These covariances, given in equations (20) and (31), are derived using a Fisher information analysis of a linear representation of the transit light curve. The key inputs are the uncertainty in each measurement of the relative flux, and the sampling rate. We have verified the accuracy of the variance and covariance estimates derived from the piecewise linear light curve with a numerical Fisher analysis of a more realistic (nonlinear) light-curve model and with a Markov chain Monte Carlo analysis of idealized data.

We focused on a particular parameterization of this piecewise linear light curve that we believe to be most useful. The parameters are the midtransit time $\left(t_{c}\right)$, the out-of-transit flux $\left(f_{0}\right)$, the flux decrement during the full phase of the transit $(\delta)$, the duration of ingress or egress $(\tau)$, and the duration between the midpoint of ingress and the midpoint of egress $(T)$. This set is observationally intuitive and gives simple analytic formulae for variances and covariances. The exact parameter definitions are provided in equations (8), (9), and (10) in terms of the normalized impact parameter, stellar and planetary radii, the semimajor axis, and the orbital period. Inverse mappings to more physical parameters are provided in equations (12), (13), and (14). The analytic covariance matrix is given in equation (20), and the analytic correlation matrix is given in equation (24). Some quick-and-dirty (but still rather accurate) expressions for the parameter uncertainties, for the case in which the planet is small, the out-oftransit flux is known precisely, and limb darkening is negligible, are given as

$$
\begin{aligned}
\sigma_{t_{c}} & =Q^{-1} T \sqrt{\theta / 2} \\
\sigma_{\tau} & \approx Q^{-1} T \sqrt{6 \theta} \\
\sigma_{T} & \approx Q^{-1} T \sqrt{2 \theta} \\
\sigma_{\delta} & \approx Q^{-1} \delta,
\end{aligned}
$$

where $\theta \equiv \tau / T$ is the ratio of the ingress or egress duration to the total duration, and $Q \equiv(\Gamma T)^{1 / 2}(\delta / \sigma)$ is the total signal-to-noise ratio of the transit in the small-planet limit (see eq. [19]).

We investigated the applicability of these results to a limbdarkened brightness profile, in which the true light curve is not as well described by a piecewise linear function. We found that the analytic formulae underestimate some of the variances and covariances by a factor of a few, for a typical degree of limb darkening at optical wavelengths. Significant improvements to covariance estimates in the limb-darkened case may be made by redefining the depth parameter as a function of the darkening coefficient and impact parameter as in equation (28). Unfortunately, no closed-form mapping to more physical parameters exists with this choice, and therefore, most of the appeal of the analytic treatment is lost.

Quantities that are derived in part or in whole from the transit light curve (such as the stellar mean density or exoplanet surface gravity) are provided in terms of the suggested parameter set. In Table 2, uncertainties propagated from the covariance estimates for these quantities are provided with simple analytic formulae. In Table 3, covariance elements relevant to the uncertainties in 
Table 2 are given for the case in which the planet is small and the out-of-transit flux is known precisely. This allows the uncertainty in a given physical parameter to be predicted in advance of any data, bypassing the need for time-consuming simulations. For transit surveys, these formulae may also be useful in giving closed-form expressions for the expected distributions for some of the key properties of a sample of transiting planets.

In $\S 6$, with the tools provided, we approach the question of what parameter sets are best suited to numerical parameter estimation codes. This question depends both on the level of parameter correlation and the behavior of any a priori likelihood functions. We advocated a parameter set that has the virtue of both weak correlation and essentially uniform a priori expectations; specifically, the parameters are the midtransit time, the out-oftransit flux, the ratio of planetary to stellar radii $\left(R_{p} / R_{\star}\right)$, the normalized impact parameter, and the duration between the midpoint of ingress and the midpoint of egress. Figure 13 graphically describes the parameter correlations, while equation (36) gives the a priori probability distribution. Finally, two parameter choices are given that are less intuitive than the suggested set but that provide smaller correlations, depending on information that may be inferred or guessed prior to analysis. Correlations may be tuned to zero with the second parameter choice for a nongrazing transit and an estimate of $R_{p} / R_{\star}$. The resulting correlation matrices for both parameter choices are given in equations (37) and (39). Lower correlations relate directly to more efficient data fitting, as demonstrated by reduced correlation lengths with a Markov chain Monte Carlo method.

We thank Philip Nutzman for helpful comments on an early version of this draft and, in particular, for pointing out the consequences of the singularity in equation (34). We also thank the referee for helpful comments and for suggesting the Markov chain technique for use with the parameter choices in equation (39). Sara Seager and Paul Joss also provided helpful comments. We are grateful for support from the William S. Edgerly Innovation Fund and from NASA grant HST-GO-11165 from the Space Telescope Science Institute, which is operated by the Association of Universities for Research in Astronomy, Inc., under NASA contract NAS5-26555.
Agol, E., Steffen, J., Sari, R., \& Clarkson, W. 2005, MNRAS, 359, 567

Alonso, R., et al. 2004, ApJ, 613, L153

Bakos, G. Á., et al. 2007, ApJ, 671, L173

Barnes, J. W. 2007, PASP, 119, 986

Brown, T. M., Charbonneau, D., Gilliland, R. L., Noyes, R. W., \& Burrows, A. 2001, ApJ, 552, 699

Burke, C. J. 2008, ApJ, 679, 1566

Burke, C. J., et al. 2007, ApJ, 671, 2115

Charbonneau, D., Brown, T. M., Burrows, A., \& Laughlin, G. 2007, Protostars and Planets V, ed. B. Reipurth, D. Jewitt, \& K. Keil (Tucson: Univ. Arizona Press), 701

Claret, A. 2000, A\&A, 363, 1081

Deming, D., et al. 2007, ApJ, 667, L199

Ford, E. B. 2005, AJ, 129, 1706 2006, ApJ, 642, 505

Ford, E. B., \& Holman, M. J. 2007, ApJ, 664, L51

Ford, E. B., Quinn, S. N., \& Veras, D. 2008, ApJ, 678, 1407

Gillon, M., et al. 2007, A\&A, 471, L51

Giménez, A. 2007, A\&A, 474, 1049

\section{REFERENCES}

Gould, A. 2003, preprint (astro-ph/0310577)

Heyl, J. S., \& Gladman, B. J. 2007, MNRAS, 377, 1511

Holman, M. J., \& Murray, N. W. 2005, Science, 307, 1288

Holman, M. J., et al. 2006, ApJ, 652, 1715

Knutson, H. A., et al. 2007, Nature, 447, 183

Mandel, K., \& Agol, E. 2002, ApJ, 580, L171

Miralda-Escude, J. 2002, ApJ, 564, 1019

Murray, C. D., \& Dermott, S. F. 2000, Solar System Dynamics (Cambridge: Cambridge Univ. Press)

Pál, A. 2008, MNRAS, 390, 281

Protopapas, P., Jimenez, R., \& Alcock, C. 2005, MNRAS, 362, 460

Richardson, L. J., Harrington, J., Seager, S., \& Deming, D. 2006, ApJ, 649, 1043 Sato, B., et al. 2005, ApJ, 633, 465

Seager, S., \& Mallén-Ornelas, G. 2003, ApJ, 585, 1038

Southworth, J. 2008, MNRAS, 386, 1644

Tegmark, M., et al. 2004, Phys. Rev. D, 69, 103501

Winn, J. N., Holman, M. J., \& Fuentes, C. I. 2007, AJ, 133, 11

Winn, J. N., et al. 2005, ApJ, 631, 1215 